\title{
Life and Demise of Intrathermocline Mesoscale Vortices
}

\author{
TIMOUR RADKO AND CASSANDRA SISTI \\ Department of Oceanography, Naval Postgraduate School, Monterey, California
}

(Manuscript received 6 March 2017, in final form 8 October 2017)

\begin{abstract}
This study is focused on finescale dissipation mechanisms of intrathermocline mesoscale vortices exemplified by meddies, large anticyclonic salt lenses of Mediterranean origin commonly observed in the lower North Atlantic thermocline. High-resolution numerical experiments are diagnosed to quantify the rates of temperature and salinity $(T, S)$ dispersion in salt lenses and to determine the relative contribution of various mixing processes in the decay of their thermohaline signatures. This study finds, in agreement with observations, that meddies dissipate on the characteristic time scale of several years and that their ultimate disintegration can be attributed to thermohaline interleaving driven by double-diffusive mixing. Mechanically generated turbulence, on the other hand, tends to suppress the interleaving and therefore has an adverse net effect on eddy dispersion. It is found that the dispersion properties of static lenses, characterized by density-compensated $T-S$ patterns, and their rapidly rotating counterparts are dramatically different.
\end{abstract}

\section{Introduction}

Mesoscale intrathermocline eddies play an essential role in transferring heat, salinity, biogeochemical tracers, and momentum in large-scale flows (e.g., Armi et al. 1989; Carton 2001; Ambar et al. 2002; Sokolovskiy and Verron 2014). Nevertheless, physical mechanisms controlling their longevity and coherence have not been fully explained (Ruddick et al. 2010; Song et al. 2011). Several observational studies (Ruddick and Hebert 1988; Hebert et al. 1990; Ruddick et al. 2010) have implicated lateral intrusions driven by double-diffusive mixing (Stern 1967) in the ultimate disintegration of coherent intrathermocline vortices. Although this suggestion is plausible, observational limitations generally preclude the unambiguous confirmation of the proposed link between eddy dispersion and thermohaline interleaving. Equally challenging is the quantification of the associated mixing characteristics on the basis of field measurements, motivating a numerical approach.

While the linear theory of thermohaline intrusions is relatively well developed and fully understood-see, for example, the review by Ruddick and Kerr (2003) - their mixing properties and the potential impact on large-scale structures have not been precisely quantified yet. The reported estimates of lateral eddy diffusivities associated

Corresponding author: Timour Radko, tradko@nps.edu with interleaving vary by orders of magnitude (Ruddick and Richards 2003; Radko 2013). The existing evidence suggests that interleaving is heavily influenced by various environmental characteristics, including rotation, baroclinicity, large-scale shears, and ambient turbulence (May and Kelley 1997; Kuzmina and Zhurbas 2000; Smyth 2008; Smyth and Ruddick 2010). However, a more systematic analysis is required to determine the specific relations between intrusive mixing and large-scale environmental parameters. These uncertainties imply that, at present, definitive answers regarding the interaction between mesoscale vortices and intrusions could only be provided by models in which interleaving is fully resolved rather than parameterized.

The requirement to simulate interleaving in a mesoscale eddy presents a formidable computational challenge. The model has to resolve a wide range of vertical scales, from the intrusion thickness $(\sim 10 \mathrm{~m})$ to the basin depth $(\sim 2000 \mathrm{~m})$. To represent an effectively isolated vortex, the horizontal domain size should greatly exceed the eddy dimensions. Typical life cycles of coherent oceanic vortices cannot be captured unless simulations are extended for time periods of several years. Computational capabilities have only recently reached the level of resolving such a wide range of spatial and temporal scales. To the best of our knowledge, the current investigation presents the first intrusion-resolving numerical simulation of an intrathermocline mesoscale eddy. 
A prototype model for this study is based on properties of meddies, North Atlantic salt lenses containing water masses of Mediterranean origin. Meddies represent an appealing subject for the dispersion analysis because they are generally far separated from the sea surface and topography, making it possible to clearly isolate the interior sources of mixing. Another incentive to focus our inquiry on meddies is provided by a substantial body of observations (McDowell and Rossby 1978; Armi et al. 1988; Pingree and Le Cann 1993; Prater and Sanford 1994; Tychensky and Carton 1998) readily available for the comparison with numerical results. In particular, the most appropriate modeling prototype for our study is Meddy Sharon, which has already been the subject of several data-based investigations (Hebert et al. 1990; Ruddick 1992; Ruddick et al. 2010) into the role of thermohaline interleaving in its ultimate dispersion.

Another interesting technical challenge in modeling intrathermocline lenses concerns the choice of the initial state. Observed oceanic vortices are commonly described using Gaussian patterns of temperature $T$, salinity $S$, and rotation rate. However, the intrathermocline Gaussian vortices in the parameter range of Meddy Sharon are strongly unstable (Sutyrin and Radko 2016). Their temporal evolution is characterized by the fragmentation of the original circular vortex into smaller and more intense coherent structures. The unrealistically rapid disintegration of Gaussian vortices in the regime of interest precludes their utilization in studies of the long-term dissipation of coherent eddies. This complication is addressed in the present work by using the recently developed stabilizing procedure of Sutyrin and Radko (2016). The proposed algorithm involves the modification of isopycnal potential vorticity (PV) gradients and the subsequent iterative reconstruction of the vortex structure.

The numerical experiments in this study are generally consistent with field-based inferences. We confirm the possibility of substantial meddy erosion on the time scale of 2-3 years caused by lateral intrusion-induced mixing. The lateral diffusivities diagnosed from our simulations fall within the range of values inferred for Meddy Sharon on the basis of microstructure measurements and observed erosion rates (Hebert et al. 1990; Ruddick et al. 2010). At the same time, the flexibility of a modeling approach yields additional physical insights that would be difficult, if not impossible, to attain based on observations alone. In particular, we show that, somewhat counterintuitively, increasing background turbulence levels adversely affects meddy dispersion by suppressing the interleaving. Major differences are found between the dynamics and dispersion properties of static lenses, characterized by density-compensated $T-S$ patterns, and their rapidly rotating counterparts.
This dissimilarity underscores the inherent limitations of modeling studies of interleaving in quiescent background states. Finally, it should be emphasized that while the key physical processes at play are illustrated here on the specific example of meddies, the broader implications of our findings are much more fundamental and far reaching. It is our belief that this study may provide important clues to one of the most fundamental and long-standing problems in physical oceanography, namely, the link between the basin-scale forcing of the ocean by air-sea fluxes and the ultimate dissipation of energy and thermal variance at the microscale.

The paper is organized as follows: In section 2, we describe the configuration of our numerical model and the algorithm used to construct dynamically stable initial states. Section 3 presents a series of simulations that reveal typical evolutionary patterns of intrathermocline vortices. These simulations are diagnosed to analyze the meddy erosion rates and to determine effective lateral diffusivities associated with interleaving. In section 4 , we identify and discuss some major differences between the dynamics of rapidly rotating meddies and their density-compensated static counterparts. In section 5, we consider various alternative meddy models and, in particular, extend our calculations to dynamically unstable vortices. We summarize the results and draw conclusions in section 6 .

\section{Formulation}

The first step in developing a consistent meddy model was the design of the initial state. The model had to meet two critical requirements: (i) the initial flow pattern should be similar to the observed structures and (ii) it should be dynamically stable. The latter condition poses a curious conundrum in the theory of isolated vortices (e.g., Dewar and Killworth 1995; Dewar et al. 1999; Benilov 2005; among many others). The longevity of coherent mesoscale vortices in the ocean is well documented. Meddies are known to persist for years, making hundreds of revolutions during their life cycles. The majority of theoretical vortex models assume the Gaussian pattern of angular velocity (e.g., Beckers et al. 2001; Benilov and Flanagan 2008). Such a model fits oceanic and laboratory vortices reasonably well (e.g., Sokolovskiy and Verron 2014). However, several stability analyses and simulations suggest that the Gaussian vortex, otherwise seemingly consistent with observations, could be unstable. In some regions of the parameter space the instability is relatively mild (e.g., Nguyen et al. 2012; Yim et al. 2016). However, simulations with Gaussian vortices in the parameter range of Meddy Sharon (Sutyrin and Radko 2016) reveal their strong instability, resulting in the rapid fragmentation of the 
initial states. This instability precludes the utilization of the Gaussian model as the meddy prototype in dispersion analyses.

\section{a. Stabilization of mesoscale vortices}

A relatively simple prescription for constructing dynamically stable meddy models was recently suggested by Sutyrin and Radko (2016). Exploiting the analogy with the celebrated Rayleigh (1879) instability condition for parallel flows (Dritschel 1988), the destabilization of Gaussian vortices was attributed to the presence of opposing radial $\mathrm{PV}$ gradients along isopycnal surfaces. The proposed stabilizing procedure therefore involves eliminating negative radial isopycnal PV gradients and iteratively reconstructing the flow field. While the resulting PV-corrected vortices are structurally similar to their Gaussian counterparts, they proved to be dramatically more robust and persistent, capable of retaining their axisymmetric structure for decades in simulations. The PV-corrected Gaussian vortices bear closer dynamic similarity to observed meddies, in which PV tends to monotonically increase with the distance from the axis of rotation (e.g., Prater and Sanford 1994). The monotonicity of the PV distribution in the oceanic intrathermocline vortices (e.g., Elliott and Sanford 1986; D'Asaro 1988; Paillet et al. 2002) is likely to be the key reason for their relative stability and longevity.

The Sutyrin-Radko stabilizing procedure was utilized in the present study as well. The azimuthal velocity distribution in the primary vortex was represented by the Gaussian model:

$$
v_{\theta}(r, z)=-A r \exp \left[-\frac{r^{2}}{r_{0}^{2}}-\frac{\left(z-z_{0}\right)^{2}}{h^{2}}\right],
$$

where $A=4.5 \times 10^{-6} \mathrm{~s}^{-1}, r_{0}=30 \mathrm{~km}, z_{0}=-1000 \mathrm{~m}$, and $h=400 \mathrm{~m}$. The expression in (1) is written in the cylindrical coordinate system $(r, z)$, where $r=\sqrt{x^{2}+y^{2}}$ is the distance from the meddy axis, and the origin of the vertical coordinate $z$ is at the sea surface. The density distribution conformed to the velocity pattern [(1)] through cyclogeostrophic and hydrostatic balances. The meddy was embedded in the uniform background vertical temperature and salinity gradients of $T_{\mathrm{bg} \_\mathrm{z}}=$ $0.008^{\circ} \mathrm{Cm}^{-1}$ and $S_{\mathrm{bg} \_\mathrm{z}}=0.0016 \mathrm{psum}^{-1}$. The corresponding density ratio is $R_{\rho}=\left(\alpha T_{\text {bg_z }_{2}}\right) /\left(\beta S_{\text {bg } \_z_{2}}\right)=1.3$, where $\alpha$ and $\beta$ are the thermal expansion and haline contraction coefficients, respectively. This density ratio is representative of "deep Meddies" observed below 700 db (e.g., Washburn and Käse 1987).

The primary vortex [(1)] was subjected to the PVcorrecting procedure (Sutyrin and Radko 2016), and the resulting patterns of temperature $T$, density $\rho$, and azimuthal velocity $v_{\theta}$ are shown in Fig. 1 . Figures $2 \mathrm{a}$ and $2 \mathrm{~b}$
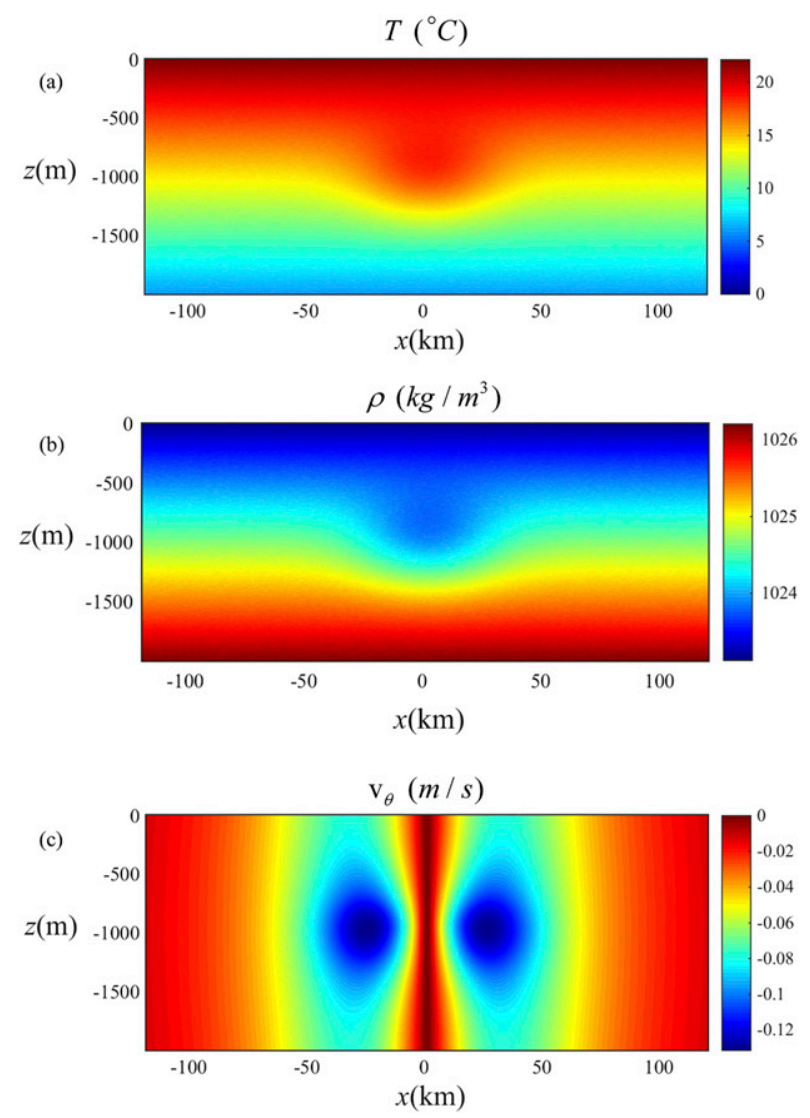

FIG. 1. The dynamically stable state used for the initialization of numerical simulations. The vertical $(x, z)$ sections of (a) temperature, (b) density, and (c) azimuthal velocity across the meddy center are shown.

present the distribution of PV (normalized by its background value) along the isopycnal surfaces in the original Gaussian (Fig. 2a) and in the PV-corrected vortex (Fig. 2b). Potential vorticity is defined as

$$
\mathrm{PV}=(f \mathbf{k}+\nabla \times \mathbf{v}) \cdot \nabla \rho,
$$

where $\mathbf{v}=(u, v, w)$ is the velocity vector, and $\mathbf{k}$ is the vertical unit vector. While the primary vortex is characterized by the reversal of its isopycnal potential vorticity gradients, the PV distribution in the stabilized vortex is monotonic. The elimination of rapidly amplifying dynamic instabilities associated with the PV reversal made it possible to focus on much slower finescale dissipation mechanisms. It is interesting that the velocity pattern of the PV-corrected vortex remains qualitatively similar to the Gaussian model (Fig. 2c). The relative rms difference between the azimuthal velocity in the PV-corrected vortex and the Gaussian fit to it is only $18 \%$. The maximal azimuthal velocity, however, increases by a factor of 2.3 relative to that in its Gaussian progenitor [(1)]. 
(a)

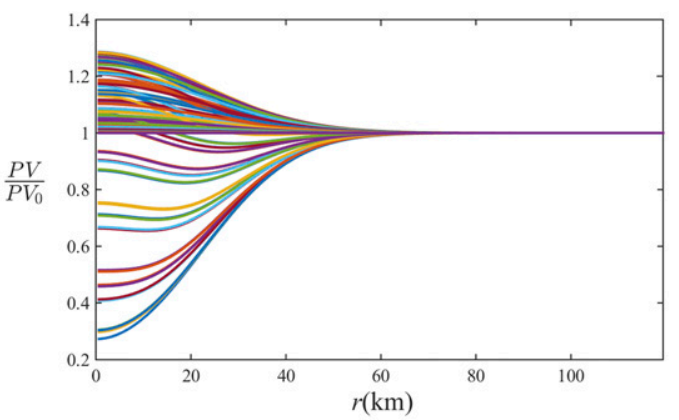

(b)

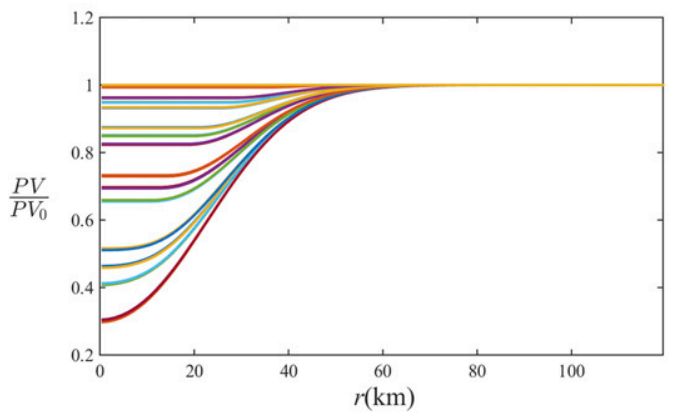

(c)

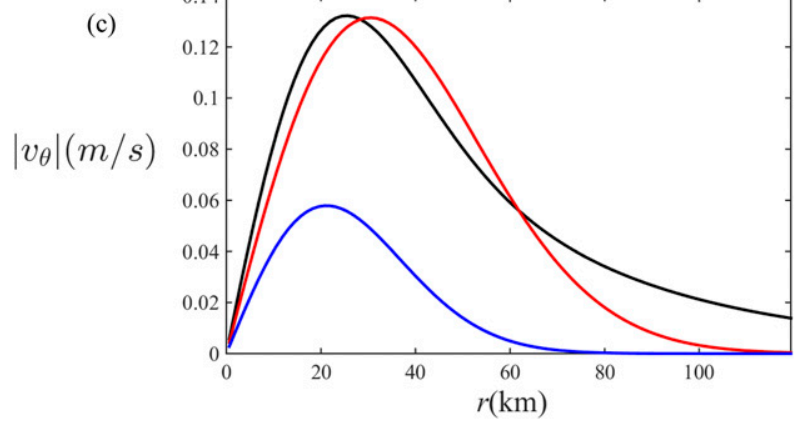

FIG. 2. The radial distribution of potential vorticity along isopycnal surfaces, normalized by the far-field value in (a) the original Gaussian vortex and (b) its stable PV-corrected counterpart. The stabilizing procedure eliminates the regions with negative radial PV gradients. (c) The radial profiles of azimuthal velocity $\left|v_{\theta}\right|$ at $z=-1000 \mathrm{~m}$ for the PV-corrected vortex (black), its Gaussian progenitor [(1)] (blue), and the best fit of the Gaussian profile to the PV-corrected vortex (red).

\section{b. Physical interpretation of thermohaline interleaving}

While the adjustment of PV gradients precluded relatively large-scale dynamic instabilities, the vortex remained susceptible to thermohaline interleaving, the main subject of our investigation. To make our discussion selfcontained, we now briefly review the conventional interpretation of thermohaline interleaving (Stern 1967; Turner 1978; Ruddick and Kerr 2003). The schematic in Fig. 3a represents a two-component fluid, which is susceptible to fingering convection and stratified both vertically and horizontally. Consider a harmonic perturbation with wave fronts that are only slightly tilted relative to

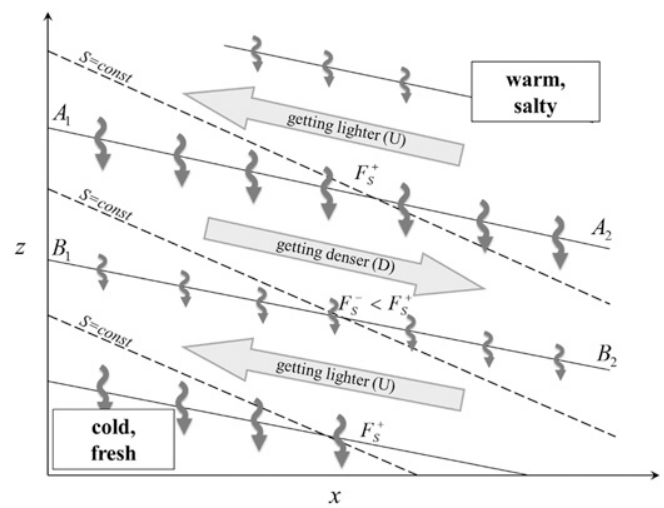

(b)

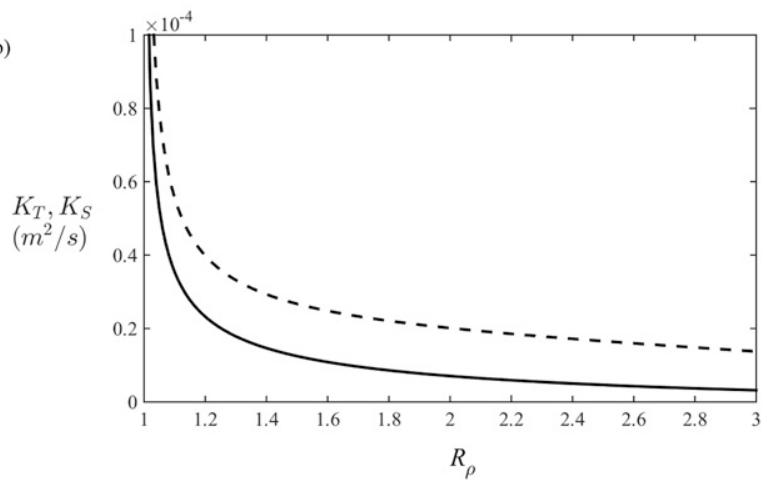

FIG. 3. (a) Schematic diagram illustrating development of thermohaline intrusions (from Radko 2013). (b) Diffusivities of temperature (solid) and salinity (dashed) used to parameterize finger-induced mixing as a function of the density ratio.

the horizontal plane; the slope of intrusions is less than the slope of isohalines but has the same sign. This perturbation enhances the vertical gradients of temperature and salinity along some wave fronts $A_{1} A_{2}$ and reduces them along others $B_{1} B_{2}$. The elevated vertical gradients imply higher finger-induced fluxes of heat and salt. As a result, the region located below the high-gradient zone $A_{1} A_{2}$ and above the weak-gradient front $B_{1} B_{2}$ becomes the site of convergence for both salinity and temperature fluxes. If fluxes of salinity contribute to diapycnal density transport more than temperature fluxes, as expected for salt fingering (e.g., Radko 2013), then the water mass located between fronts $A_{1} A_{2}$ and $B_{1} B_{2}$ becomes denser and slides downward. The density-induced flow advects fresher and colder water into this region, which further intensifies the $T-S$ gradients along the highgradient front $A_{1} A_{2}$ and weakens the gradients along the low-gradient front $B_{1} B_{2}$. The resulting positive feedback loop reinforces the initial perturbation, causing the amplification of intrusions. It should be emphasized that the mechanism of interleaving instability illustrated in Fig. 3a depends critically on the substantial difference between the temperature and salinity diffusivities. Since the $T-S$ 
perturbations in the growing intrusion (Fig. 3a) are anticorrelated with horizontal velocity, we expect interleaving to produce substantial lateral downgradient $T-S$ fluxes. One of the key objectives of our study is the quantification of this intrusion-driven lateral mixing based on a suite of numerical experiments.

\section{c. Numerical model}

Simulations in the present study were performed using the Massachusetts Institute of Technology General Circulation Model (MITgcm). MITgcm solves the governing (incompressible Navier-Stokes) equations by the finitevolume method (Marshall et al. 1997a,b). All presented experiments were carried out using a computational domain of $240 \mathrm{~km} \times 240 \mathrm{~km} \times 2 \mathrm{~km}$, which was resolved by a uniform mesh with $192 \times 192 \times 2000$ grid points in $x, y$, and $z$, respectively. Simulations were performed on

$$
\left\{\begin{array}{l}
K_{S}=k_{T}\left(\frac{a_{S}}{\sqrt{R_{\rho}-1}}-b_{S}\right) R_{\rho}, \\
K_{S}=K_{T}=0
\end{array}\right.
$$

where $k_{T}=1.38 \times 10^{-7} \mathrm{~m}^{2} \mathrm{~s}^{-1}$ is the molecular diffusivity of temperature, $a_{S}=135.7, b_{S}=62.75$, and $R_{0}=$ 5.67. Parameter $\gamma$ in (3) represents the heat-salt buoyancy flux ratio, which is kept at the default value of $\gamma=0.7$ assumed by MITgcm. Direct numerical simulations indicate that the flux ratio weakly varies with the density ratio (Radko and Smith 2012). However, the $\gamma=$ const model suppresses the layering instability (Radko 2003; Stellmach et al. 2011), which is caused by the variation in flux ratio, thus making it possible to focus our investigation exclusively on the effects of thermohaline interleaving. The assumed fingering diffusivities $\left(K_{T}, K_{S}\right)$ are plotted as a function of density ratio in Fig. 3b. For $R_{\rho} \sim 1.3$, which is representative of young deep meddies, thermal diffusivity is $K_{T} \sim 2 \times$ $10^{-5} \mathrm{~m}^{2} \mathrm{~s}^{-1}$. This value is broadly in agreement with observational estimates, such as $K_{T} \sim 3 \times 10^{-5} \mathrm{~m}^{2} \mathrm{~s}^{-1}$ inferred by Ruddick et al. (2010) for Meddy Sharon. We also note that the diffusivity of salinity in Fig. 3b significantly exceeds that of temperature, which is essential for triggering interleaving in ocean regions stratified in the finger-favorable sense (e.g., Stern 1967).

\section{Evolutionary patterns and dissipation rates of intrathermocline vortices}

To explore the impact of interleaving on meddies, the PV-corrected vortex in Fig. 1 was used as an initial$$
\begin{gathered}
K_{T}=\frac{\gamma K_{S}}{R_{\rho}} \quad \text { for } \quad 1<R_{\rho}<R_{0} \\
\text { for } \quad R_{\rho}>R_{0}
\end{gathered}
$$

the $f$ plane $\left(f=10^{-4} \mathrm{~s}^{-1}\right)$ using the nonhydrostatic version of MITgcm. Periodic boundary conditions were assumed in $x$ and $y$, the free-slip boundary condition was applied at the bottom, and the model was integrated with a time step of $\Delta t=100 \mathrm{~s}$. To ensure numerical stability of the model, the horizontal and vertical viscosity coefficients were set to $A_{H}=0.25 \mathrm{~m}^{2} \mathrm{~s}^{-1}$ and $A_{V}=2.5 \times 10^{-6} \mathrm{~m}^{2} \mathrm{~s}^{-1}$, respectively. Various diapycnal mixing processes in MITgcm are represented by the nonlocal K-profile parameterization (KPP) scheme (Large et al. 1994). However, the default parameterization of salt fingering in MITgcm assumes unrealistically large vertical finger-induced heat diffusivity in the regime of interest $\left(K_{T} \sim 10^{-3} \mathrm{~m}^{2} \mathrm{~s}^{-1}\right.$ for $\left.R_{\rho} \sim 1.3\right)$. Therefore, for this study, the Large et al. (1994) fingering model was replaced by a more recent closure (Radko and Smith 2012) based on a suite of direct numerical simulations:

condition for a series of numerical simulations. Figure 4 presents the evolutionary pattern of the meddy over the course of 1000 days, revealing the systematic reduction of its thermal signature in time. This simulation is hereafter referred to as EXP1, and the summary of all experiments in this study is given in Table 1 . The perturbation induced by the meddy at the final stage of the experiment (Figs. 4d,h) is approximately half of its initial value. Of particular interest for our discussion is the appearance of relatively thin (10-20 m), nearly horizontal laminae in the vertical cross sections of temperature (Figs. 4a-d). These structures are interpreted as thermohaline intrusions driven by double-diffusive mixing, and they will be shown to play a critical role in meddy dispersion. To better visualize intrusions, the temperature field was first smoothed vertically with the running average interval of $20 \mathrm{~m}$. The resulting large-scale field was denoted by $T_{\mathrm{ls}}$ and the finescale component $T_{\mathrm{fs}}$ was calculated as follows:

$$
T_{\mathrm{fs}}=T-T_{\mathrm{ls}} .
$$

The finescale component $T_{\mathrm{fs}}$ is shown in Fig. 5 for $t=10^{7} \mathrm{~s}$, equivalent to 116 days of integration. Figure 5 a presents the vertical $(x, z)$ section of $T_{\mathrm{fs}}$, which indicates that intrusions are most widespread and pronounced in the lower, finger-favorable $\left(T_{\mathrm{ls}_{-} z}>0, S_{\mathrm{l} \_z}>0\right)$ part of the meddy. The three-dimensional structure of intrusions is illustrated in Fig. 5b, which shows a relatively thin 

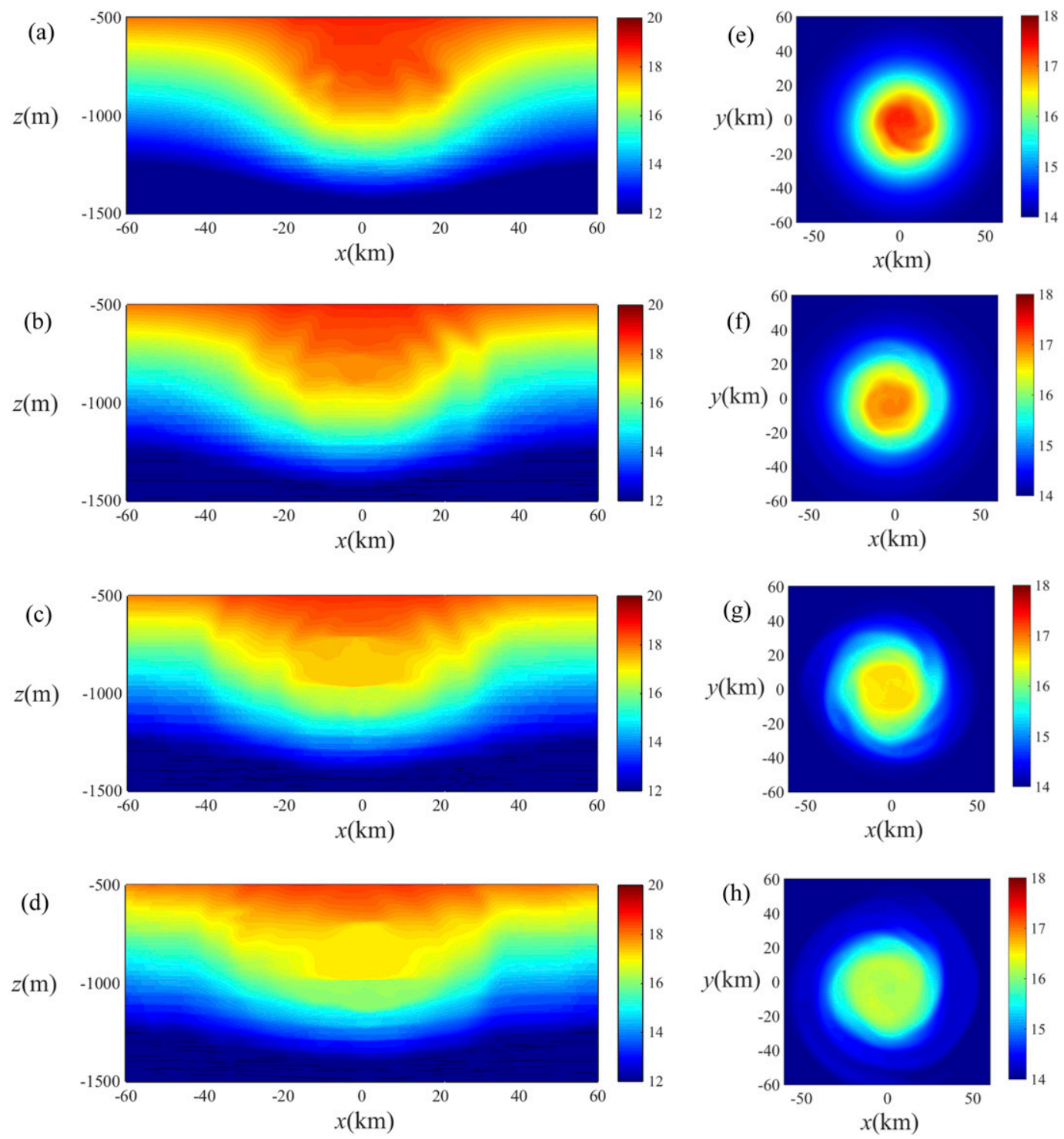

FIG. 4. Evolution of the meddy in the simulation EXP1. The vertical temperature sections $T(x, z)$ at $y=0$ for $t=($ a) 250 , (b) 500, (c) 750, and (d) 1000 days. (e)-(h) The corresponding horizontal sections $T(x, y)$ at $z=-1000 \mathrm{~m}$. Shown is the central, most active region of the computational domain $(-60<x<60 \mathrm{~km},-60<y<60 \mathrm{~km},-1500<z<-500 \mathrm{~m})$.

vertical slice $(-1100<z<-900 \mathrm{~m})$ of the meddy in its northeastern quadrant $(x>0, y>0)$. Figure $5 \mathrm{~b}$ reveals the lateral coherence of intrusions; particularly notable is their remarkable uniformity in the azimuthal direction.

To be more quantitative in describing the erosion and eventual loss of coherence of the meddy, we introduce the diagnostic variable $R_{\text {Tvar }}$ defined as follows:

$$
R_{\mathrm{Tvar}}=\frac{\left\langle\left(T-T_{\mathrm{av}}\right)^{2}\right\rangle}{\left.\left\langle\left(T-T_{\mathrm{av}}\right)^{2}\right\rangle\right|_{t=0}},
$$

where $T_{\mathrm{av}}(z)$ is the horizontally averaged temperature, and the angled brackets represent the volume average over the computational domain. As the meddy erodes, its isotherms flatten and $R_{\text {Tvar }}$ systematically reduces in time. Thus, $R_{\text {Tvar }}$ provides a convenient integral measure of the meddy's intensity relative to its initial value. The time record of $R_{\text {Tvar }}$ for simulation EXP1 (Fig. 6) is generally consistent with qualitative inferences based on Fig. 4, suggesting the meddy erosion time scale of 2-3 years. To determine the role of thermohaline interleaving in meddy dissipation, the simulation EXP1 was reproduced without engaging double-diffusive mixing (EXP2). The resulting pattern of $R_{\text {Tvar }}(t)$ for EXP2 is also presented in Fig. 6 . The outcome, however, is dramatically different. In EXP1, by $t=1200$ days the diagnostic variable reduced to 
TABLE 1. Summary of all numerical experiments presented in this study. SR16 denotes the vortex model constructed using stabilizing algorithm developed by Sutyrin and Radko (2016).

\begin{tabular}{|c|c|c|c|}
\hline Experiment & $\begin{array}{c}\text { Double } \\
\text { diffusion }\end{array}$ & $\left(K_{H}, K_{V}\right)\left(\mathrm{m}^{2} \mathrm{~s}^{-1}\right)$ & Initialization \\
\hline EXP1 & Yes & $(0,0)$ & SR16 \\
\hline EXP2 & No & $(0,0)$ & SR16 \\
\hline EXP3 & No & $(1,0)$ & SR16 \\
\hline EXP4 & No & $(3,0)$ & SR16 \\
\hline EXP5 & Yes & $\left(0,0.5 \times 10^{-5}\right)$ & SR16 \\
\hline EXP6 & Yes & $\left(0,1 \times 10^{-5}\right)$ & SR16 \\
\hline EXP7 & Yes & $\left(0,2 \times 10^{-5}\right)$ & SR16 \\
\hline EXP8 & Yes & $(0,0)$ & Quiescent \\
\hline EXP9 & Yes & $(0,0)$ & PV Gaussian $(r, z)$ \\
\hline EXP10 & Yes & $(0,0)$ & PV Gaussian $(r, \rho)$ \\
\hline EXP11 & No & $(0,0)$ & PV Gaussian $(r, z)$ \\
\hline EXP12 & No & $(0,0)$ & PV Gaussian $(r, \rho)$ \\
\hline EXP13 & Yes & $(0,0)$ & Gaussian \\
\hline EXP14 & No & $(0,0)$ & Gaussian \\
\hline
\end{tabular}

$R_{\text {Tvar }}=0.334$, whereas the corresponding value for EXP2 is $R_{\text {Tvar }}=0.953$. Thus, without interleaving, the meddy loses less than $5 \%$ of its initial intensity over the course of more than 3 years.

Given the critical role played by interleaving in meddy dissipation, it becomes highly desirable to quantify the corresponding effective lateral diffusivity $K_{H}$. While the lateral $T-S$ fluxes are readily available in the MITgcm output, their analysis proved to be ambiguous because of a peculiar technical complication. The meddy azimuthal velocities $\left(\sim 0.1 \mathrm{~m} \mathrm{~s}^{-1}\right)$ exceed the radial speed of intrusive motions $\left(\sim 10^{-4} \mathrm{~m} \mathrm{~s}^{-1}\right)$ by at least three orders of magnitude. Therefore, even a minimal departure of meddy from circular symmetry results in a substantial contamination of the radial intrusive fluxes by mesoscale advective velocities. As a result, the record of radial $T-S$ fluxes becomes dominated by temporal variability, precluding the precise evaluation of time-mean intrusive fluxes. Therefore, the lateral transport in this study was evaluated indirectly, from the integral heat budget of the meddy. We considered the cylindrical control volume $V$ with the radius corresponding to the point of largest lateral thermal gradient in the initial state (Fig. 1) along its center plane $\left(r_{\max }=23750 \mathrm{~m}\right)$ and the vertical extent based on the extrema of vertical thermal gradients along its axis $\left(z_{\text {top }}=-717 \mathrm{~m}, z_{\text {bot }}=-1283 \mathrm{~m}\right)$. The temperature budget for this time-invariant control volume takes the following form:

$$
\frac{\partial}{\partial t} \int_{V} T d V+F_{H} S_{H}+F_{\text {top }} S_{V}-F_{\text {bot }} S_{V}=0,
$$

where $S_{H}=2 \pi r_{\text {max }}\left(z_{\text {top }}-z_{\text {bot }}\right), S_{V}=\pi r_{\text {max }}^{2},\left(F_{\text {top }}, F_{\text {bot }}\right)$ are the average vertical fluxes across the upper and lower planes of the cylinder, and $F_{H}$ is the sought average radial temperature flux at $r=r_{\max }$. The formulation
(6) was applied to the simulation EXP1 and averaged over the period $200<t<400$ days. During this time interval, interleaving was already fully developed but the meddy has not significantly dissipated yet. The inferred values of the mean flux $F_{H}$ and diffusivity are as follows:

$$
F_{H}=1.25 \times 10^{-4}{ }^{\circ} \mathrm{Cm} \mathrm{s}^{-1}, \quad K_{H}=-\frac{F_{H}}{\bar{T}_{r}}=1.6 \mathrm{~m}^{2} \mathrm{~s}^{-1},
$$

where $\bar{T}_{r}$ is the mean radial temperature gradient at $r=r_{\text {max }}$ (overbars hereinafter represent large-scale averaging). This mean gradient was estimated by averaging the local radial gradients over the frontal region of the meddy defined as

$$
\frac{r_{\max }}{2}<r<\frac{3 r_{\max }}{2}, \quad z_{\mathrm{bot}}<z<z_{\mathrm{top}} .
$$

The diagnosed diffusivity (7) is in the range of observational estimates (e.g., Ruddick et al. 2010), which instills further confidence in the ability of our model to represent interleaving at the quantitative level.

The classical physical interpretation of processes ultimately leading to the lateral mixing by fully developed intrusions was given by Joyce (1977). Building on the earlier concepts developed for diapycnal mixing (Osborn and Cox 1972), Joyce examined the dominant balance realized in the conservation equation for temperature variance:

$$
\frac{\partial\left(T^{2}\right)}{\partial t}+2 T \nabla \cdot(\mathbf{v} T)=2 K_{V} T \nabla^{2} T .
$$

The temperature and velocity fields were then separated into the large/mesoscale $(\bar{T}, \overline{\mathbf{v}})$ and finescale $(\tilde{T}, \tilde{\mathbf{v}})$ components. Joyce (1977) suggested that in frontal regions, characterized by active interleaving, the dominant mesoscale balance of the temperature variance equation is between the variance production by lateral intrusioninduced displacements and its ultimate dissipation caused by microscale mixing:

$$
P=-2 \overline{\tilde{v}_{r} \tilde{T}} \frac{\partial \bar{T}}{\partial r} \approx 2 \overline{2 \tilde{K}_{V}\left(\frac{\partial \tilde{T}}{\partial z}\right)^{2}} .
$$

The assumed production-dissipation balance (10) can be expressed in terms of lateral finescale diffusivity as follows:

$$
K_{H}=\frac{\overline{\tilde{K}_{V}\left(\frac{\partial \tilde{T}}{\partial z}\right)^{2}}}{\left(\frac{\partial \bar{T}}{\partial r}\right)^{2}} .
$$


(a)

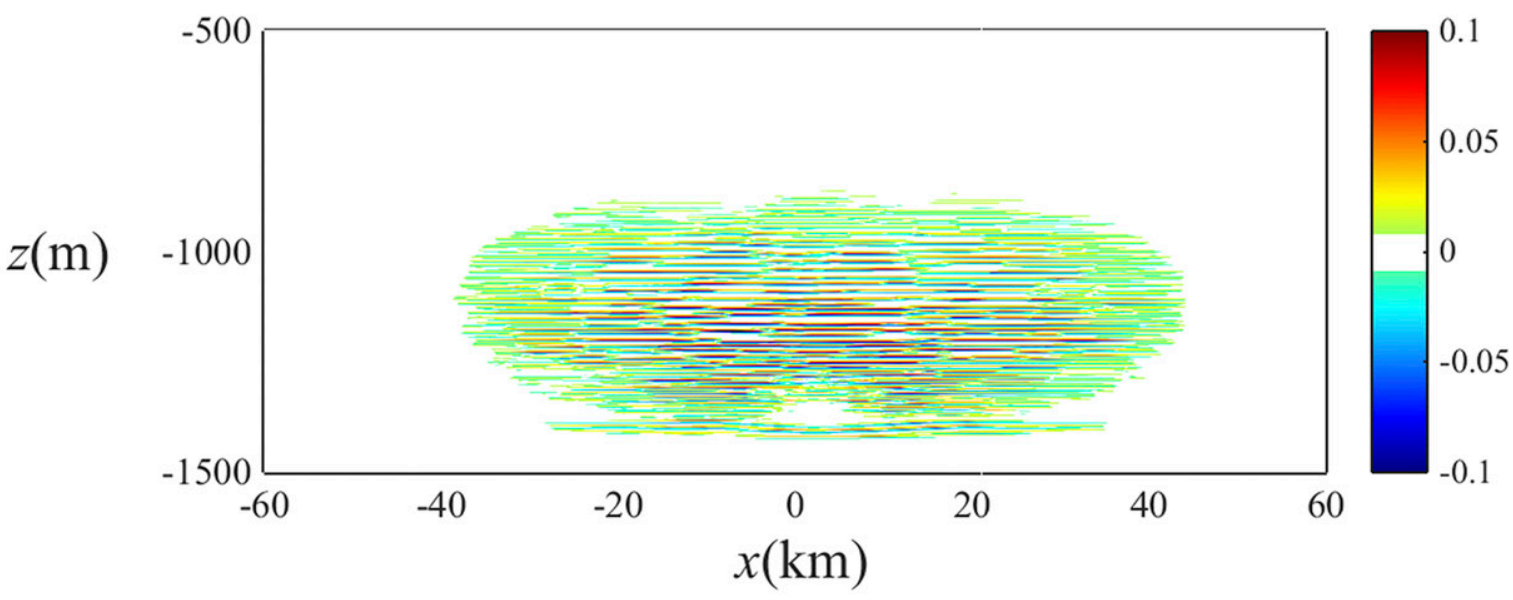

(b)

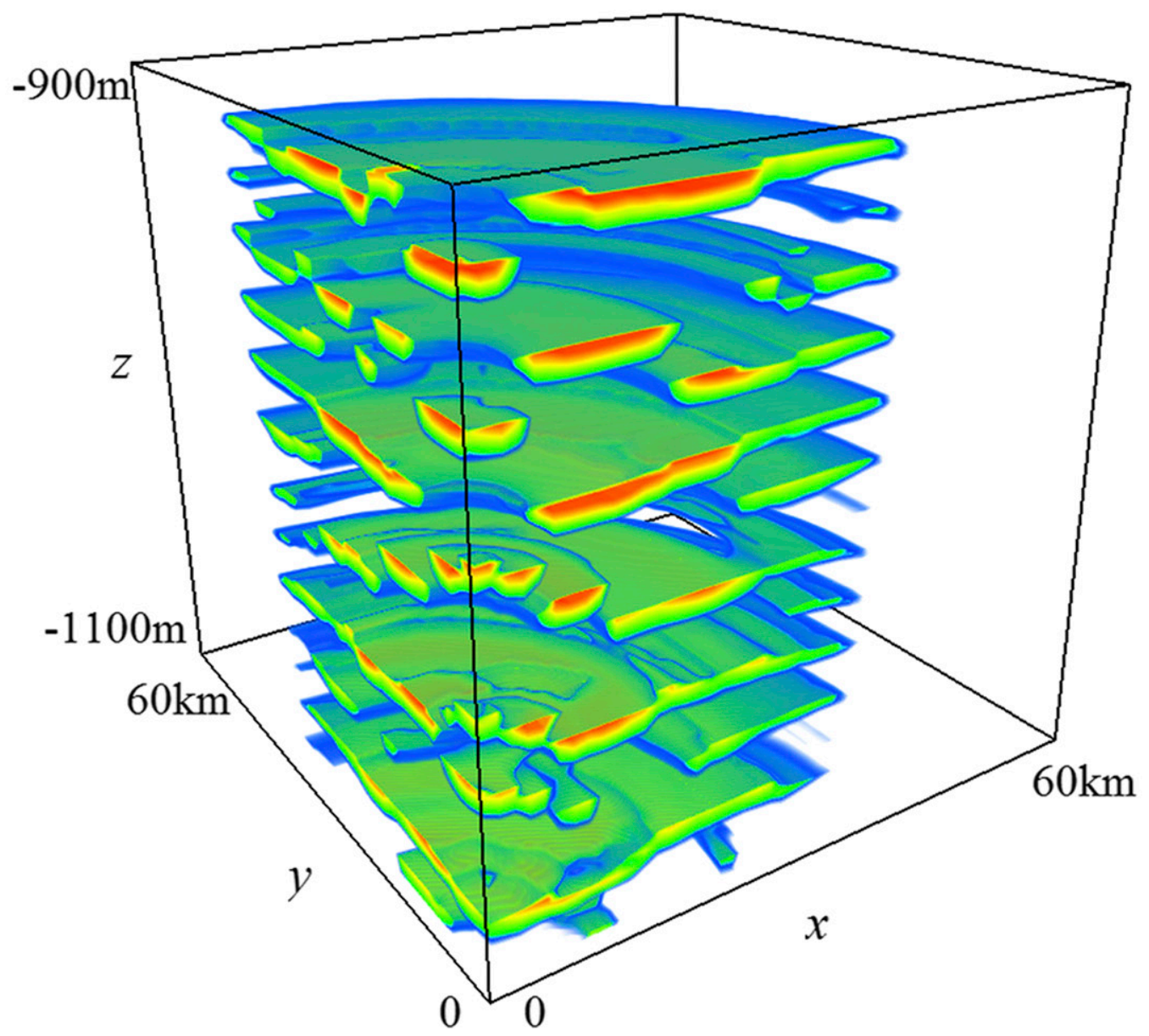

FIG. 5. Development of thermohaline intrusions in the simulation EXP1. The finescale component of the temperature field $T_{\mathrm{fs}}$ is presented at $t=10^{7} \mathrm{~s} \approx 116$ days. (a) The vertical section $T_{\mathrm{fs}}(x, z)$ at $y=0$ in the central region of the computational domain. (b) The volume-rendered image of the vertical slice $(-1100<z<-900 \mathrm{~m})$ of the meddy in its northeastern quadrant $(x>0, y>0)$. Negative values of $T_{\mathrm{fs}}$ are transparent and low (high) positive values are shown in blue (red). 


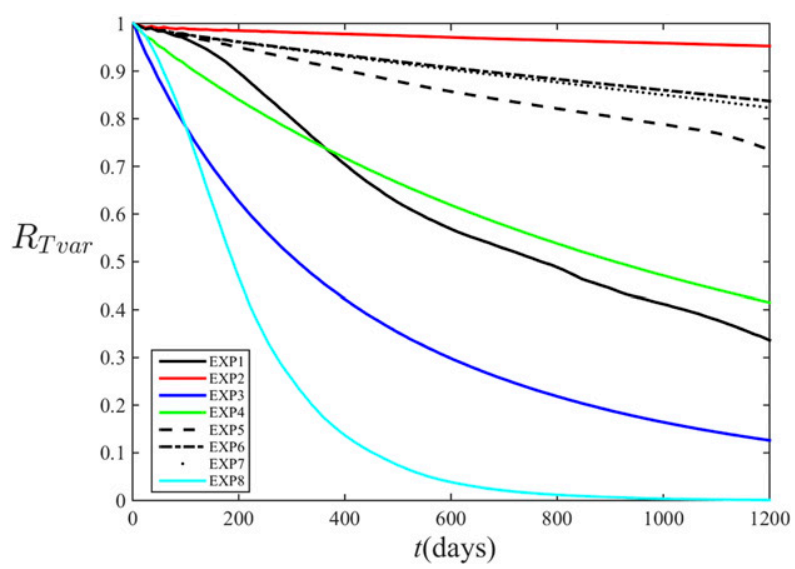

FIG. 6. The diagnostic variable $R_{\text {Tvar }}$, representing an integral measure of the meddy erosion, as a function of time for simulations EXP1-EXP8.

The application of (10) and (11) to field measurements is generally hindered by the lack of concurrent measurements of microscale diffusivities $\left(\tilde{K}_{V}\right)$ and finescale thermal gradients $(\partial \tilde{T} / \partial z)$. To surmount this complication, at least two approaches have been proposed. Joyce et al. (1978) for instance assumed a constant representative value of $\tilde{K}_{V}$ and used it to evaluate intrusive diffusivities in the Antarctic Circumpolar Front. Ruddick et al. (2010), on the other hand, expressed (10) in terms of microscale dissipation:

$$
\chi_{T}=2 k_{T}\left|\nabla T_{m}\right|^{2}
$$

where $T_{m}$ is the microscale temperature component. Using microstructure data collected in Meddy Sharon, Ruddick et al. (2010) evaluated the lateral interleaving diffusivity $K_{H}=\chi_{T} /\left[2(\partial \bar{T} / \partial r)^{2}\right]$ to be on the order of $3 \mathrm{~m}^{2} \mathrm{~s}^{-1}$.

The key advantage of modeling studies relative to their field-based counterparts is the amount of readily available information about the state of the system. In the present investigation, the knowledge of microscale diffusivities and finescale gradients at all locations makes it possible to directly test the commonly assumed balance between lateral production and vertical dissipation in the interleaving-rich areas (Joyce 1977). For that, (10) was spatially averaged over the frontal region of the meddy [(8)] and temporally averaged over the period $200<t<400$ days. This calculation suggested the following lateral intrusion-driven diffusivity [(11)]:

$$
P=1.32 \times 10^{-8}{ }^{\circ} \mathrm{Cs}^{-2}, \quad K_{H}=\frac{P}{2 \bar{T}_{r}^{2}}=1.1 \mathrm{~m}^{2} \mathrm{~s}^{-1} .
$$

The value of $K_{H}$ obtained from the productiondissipation balance [(13)] is broadly consistent with the alternative estimate in (7). The discrepancy can be readily attributed to several approximations involved in the formulation of Joyce's (1977) model. Of particular concern are (i) the inherent time dependence of mesoscale field, representing a slowly dissipating meddy, and (ii) the assumed disparity of the horizontal and vertical mesoscale production terms. Nevertheless, the agreement of (7) and (13) within 50\% implies that the essential physics of thermohaline interleaving can be adequately represented by the interplay between lateral finescale stirring and irreversible vertical mixing.

The possibility of constraining the lateral interleaving diffusivity by simulations raises the question of whether coarse-resolution models, in which intrusions are parameterized rather than resolved, could possibly capture salient features of meddy erosion. Therefore, our baseline experiment EXP1 was reproduced without explicitly incorporating double-diffusive mixing in the model formulation. Instead, the lateral eddy diffusivities of heat and salinity were assigned uniform values $K_{H}$. Two simulations of this type (EXP3 and EXP4) are presented in Fig. 6; these experiments were performed with $K_{H}=$ $1 \mathrm{~m}^{2} \mathrm{~s}^{-1}$ and $K_{H}=3 \mathrm{~m}^{2} \mathrm{~s}^{-1}$. The erosion records $R_{\text {Tvar }}(t)$ for EXP3 and EXP4 are qualitatively consistent with the intrusion-resolving run EXP1 $\left(K_{H}=1.6 \mathrm{~m}^{2} \mathrm{~s}^{-1}\right)$. The $K_{H}=1 \mathrm{~m}^{2} \mathrm{~s}^{-1}$ simulation (EXP3) generally follows the pattern of EXP1, whereas the $K_{H}=3 \mathrm{~m}^{2} \mathrm{~s}^{-1}$ experiment (EXP4) overestimates the erosion rate by a factor of 3 . The general similarity of the erosion patterns in EXP1, EXP3, and EXP4 holds the promise of developing simple closure models for lateral intrusion-driven mixing. It should be emphasized, however, that such parameterized models are not expected to fully capture the interaction between intrusions, large-scale flows, double diffusion, and mechanically generated turbulence. Therefore, their utility may be limited to inherently qualitative descriptions.

Another key question in the intrusion theory is the potential impact of mechanically generated ambient turbulence on the intensity and mixing characteristics of interleaving (e.g., Zhurbas and Oh 2001; Smyth and Ruddick 2010). This issue was addressed by reproducing our baseline experiment (EXP1) by incorporating, in addition to double-diffusive mixing, finite-uniform values of background diapycnal diffusivity $K_{\text {turb }}=0.5 \times 10^{-5}$, $1 \times 10^{-5}$, and $2 \times 10^{-5} \mathrm{~m}^{2} \mathrm{~s}^{-1}$. These simulations are referred to as EXP5, EXP6, and EXP7, respectively, and the resulting patterns of the erosion records $R_{\text {Tvar }}(t)$ are also plotted in Fig. 6. The net influence of turbulent mixing on meddy erosion is strong and-somewhat counterintuitivelyadverse. Apparently, the enhancement of the diapycnal dispersion of thermal anomalies by turbulence is greatly outweighed by their tendency to suppress interleaving and thus to limit lateral dispersion. The effective lateral diffusivities inferred from the temperature budget [(6)] 

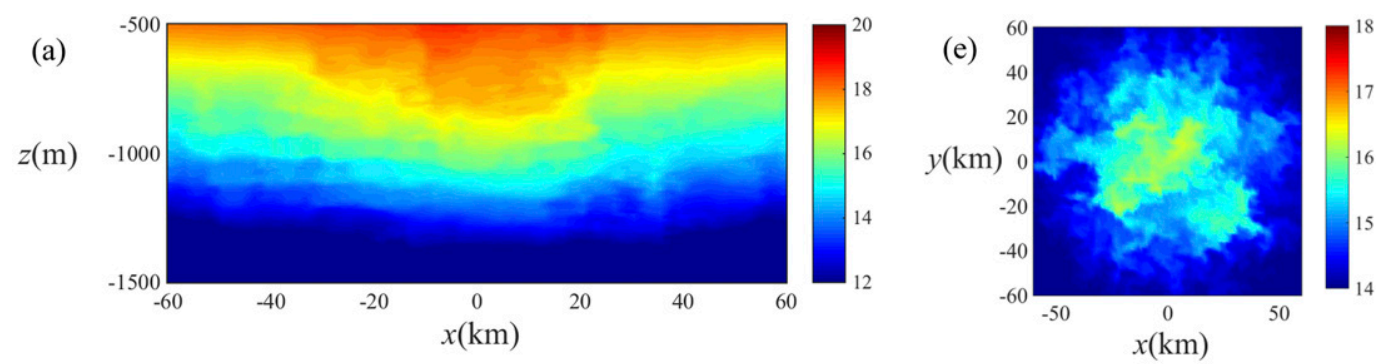

(b)
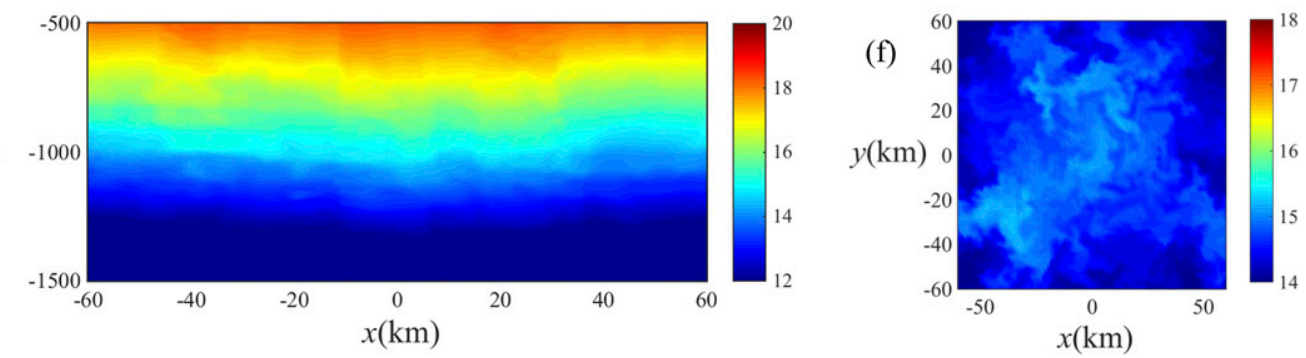

(c)
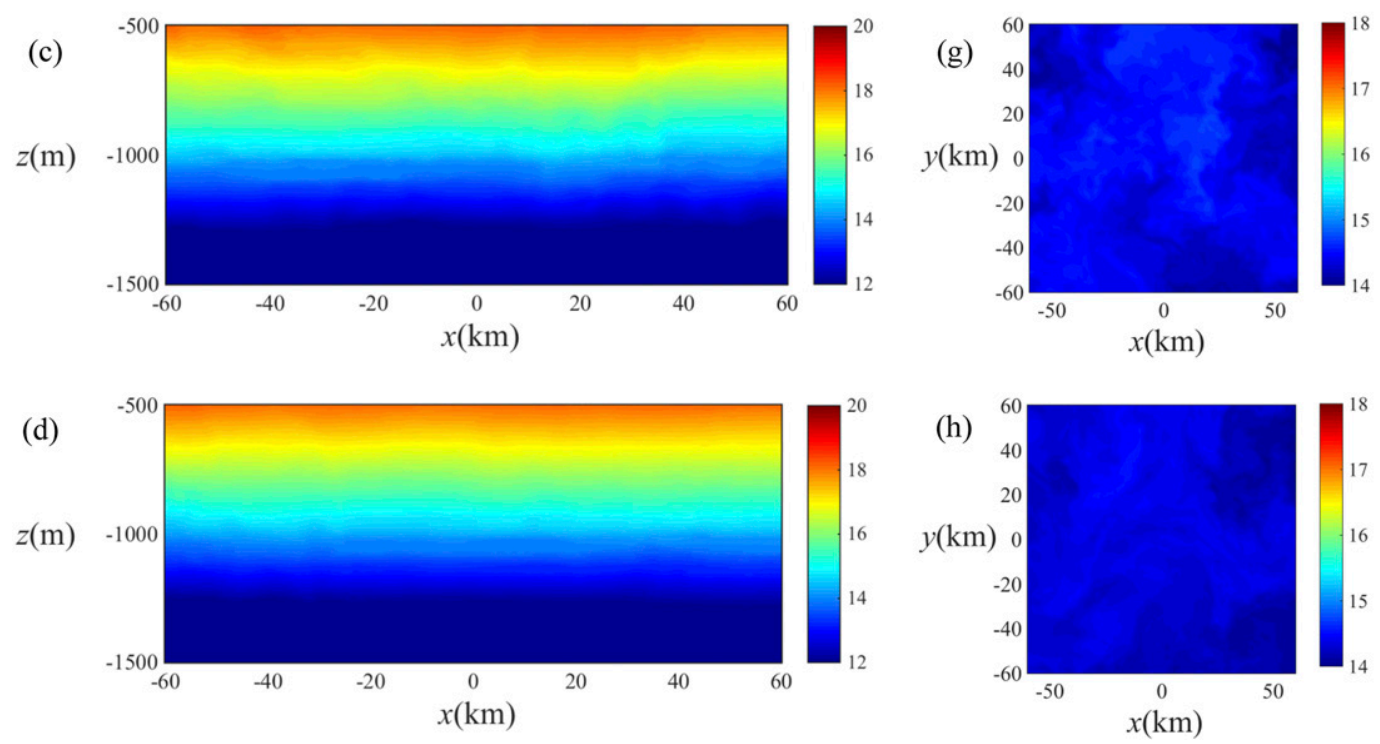

FIG. 7. As in Fig, 4, but for the density-compensated meddy (EXP8).

for EXP5-EXP7 reduce to $K_{H}=0.42,0.31$ and $0.28 \mathrm{~m}^{2} \mathrm{~s}^{-1}$, respectively. It is not clear, however, whether the homogeneous mixing model $\left(K_{\text {turb }}=\right.$ const $)$ can adequately represent the interaction between highly intermittent ocean turbulence and interleaving.

\section{Effects of vertical mesoscale shears}

Perhaps the most unexpected finding of this study concerns the controlling influence of vertical mesoscale shears on the intrusion-driven dispersion. To assess the role of baroclinicity in meddy dynamics, the baseline experiment EXP1 was compared with its static counterpart (EXP8), in which the initial state was represented by density-compensated Gaussian $T-S$ anomalies:
$\left(T_{\text {Meddy }}, S_{\text {Meddy }}\right)=\left(T_{0}, S_{0}\right) \exp \left[-\frac{r^{2}}{r_{0}^{2}}-\frac{\left(z-z_{0}\right)^{2}}{h^{2}}\right], \quad v_{\theta}=0$,

where $T_{0}=4^{\circ} \mathrm{C}$ and $S_{0}=\left(\alpha T_{0}\right) / \beta=1.07 \mathrm{psu}$. This modification resulted in a dramatic enhancement of meddy dispersion. The erosion record $R_{\mathrm{Tvar}}(t)$ for EXP8 in Fig. 6 shows that the density-compensated meddy largely dissipated within 1 year. Figure 7 presents the evolution of the temperature field in time, and the differences with the corresponding patterns in the rapidly rotating meddy of EXP1 (Fig. 4) are striking. By the end of the simulation (Figs. 7d,h) the isotherms became nearly flat, with no visible signs of any coherent remnants of the meddy. 


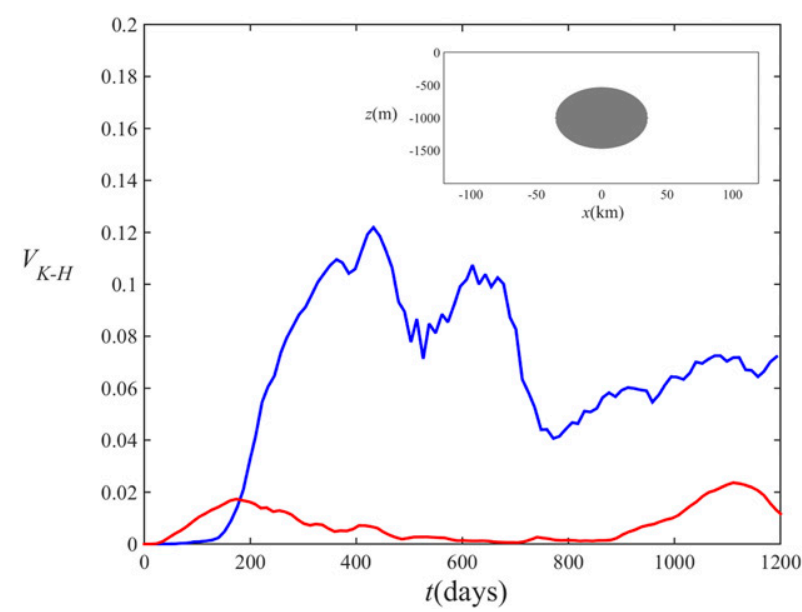

FIG. 8. The fraction of the meddy volume susceptible to largescale Kelvin-Helmholtz instabilities $V_{\mathrm{K}-\mathrm{H}}$ as a function of time. The time records of $V_{\mathrm{K}-\mathrm{H}}$ for the baroclinic, rapidly rotating meddy (EXP1) and its density-compensated counterpart (EXP8) are shown in blue and red, respectively. The shaded area in the insert indicates the operational definition of the meddy volume.

While it is natural to expect a significant impact of baroclinic shears on the intrusive dynamics (e.g., Smyth and Ruddick 2010), the magnitude of this influence is surprising and requires physical explanation. The contrasting behavior of rapidly rotating and quiescent density-compensated meddies (Figs. 4,7) is rationalized by considering the susceptibility of the two systems to Kelvin-Helmholtz (K-H) instability. According to classical instability theory (Richardson 1920; Howard 1961; Miles 1961), shear flows become unstable when the Richardson number Ri falls below the critical number of $\mathrm{Ri}_{\text {cr }}=1 / 4$. The Richardson number is defined as

$$
\mathrm{Ri}=\frac{N^{2}}{u_{z}^{2}+v_{z}^{2}},
$$

where $N=\sqrt{-(g / \rho)(\partial \rho / \partial z)}$ is the buoyancy frequency. The operational instability condition used in general circulation models often differ from the canonical result. For instance, the default condition for MITgcm to engage the interior mixing in the KPP module is $\mathrm{Ri}_{\mathrm{cr}}=0.7$. This modification reflects the possibility that the Richardson numbers evaluated on a discrete numerical mesh may not capture local minima realized in the continuously stratified ocean.

The structure of expression (15) yields an important insight into the root cause of the dissimilarity between rotating and quiescent meddies. The baroclinic meddy is characterized by strong mesoscale shears, and thus the denominator of the Richardson number can be large. Therefore, dynamic instability could be triggered by a relatively modest reduction in $N^{2}$. In the densitycompensated case (EXP8), on the other hand, mesoscale shears are nearly absent, and therefore dynamic instabilities are engaged only when the vertical density gradients are close to zero. Thus, intrusions in densitycompensated fronts can grow, unimpeded by KelvinHelmholtz instabilities, to levels that are larger than in baroclinic flows. It should be emphasized that the foregoing mechanism assumes the suppression of interleaving by relatively large-scale instabilities with dimensions comparable to the vertical extent of individual intrusions. The small-scale $(\sim 1 \mathrm{~m})$ dynamic/convective instabilities do not necessarily inhibit intrusive motions. On the contrary, these local overturns play an essential role in the dynamics of fully nonlinear interleaving (e.g., Ruddick and Kerr 2003; Mueller et al. 2007) by transporting heat and salt through the weakly stratified regions separating high-gradient fingering and diffusive interfaces. The relatively large $(\sim 20 \mathrm{~m})$ overturns, on the other hand, can effectively disrupt the coherent structure of intrusions and thus have strong adverse effects on the interleaving-driven dispersion.

The proposed hypothesis is supported by the following diagnostics (Fig. 8): For each state recorded in experiments EXP1 and EXP8, we computed the largescale distribution of the Richardson number. As previously, large-scale fields were constructed by smoothing all properties over the vertical scales of $20 \mathrm{~m}$. At each time step, we evaluated the fraction of meddy volume $V_{\mathrm{K}-\mathrm{H}}$ that is dynamically unstable according to the operational criterion used in MITgcm $(\mathrm{Ri}<0.7)$. The total meddy volume for this calculation was defined as the region where the initial temperature perturbation exceeds $1^{\circ} \mathrm{C}$, as indicated in the insert on Fig. 8. The comparison of the resulting time series of $V_{\mathrm{K}-\mathrm{H}}(t)$ in Fig. 8 reveals the striking difference in the extent of susceptibility of quiescent and rapidly rotating meddies to Kelvin-Helmholtz instability. In the baroclinic meddy, a significant fraction of its volume (up to $13 \%$ ) is affected by relatively large-scale $(\sim 20 \mathrm{~m})$ overturns. In the density-compensated experiment, $V_{\mathrm{K}-\mathrm{H}}$ is significantly less-never exceeding 3\%-which implies a much more favorable environment for initiating and sustaining active interleaving.

\section{Alternative models}

To ensure that our conclusions are sufficiently general and not model dependent, the numerical simulations in section 3 were reproduced using different initial states. The first example (EXP9) was initiated using an explicit (Gaussian) pattern of PV in the cylindrical $(r, z)$ coordinate system: 

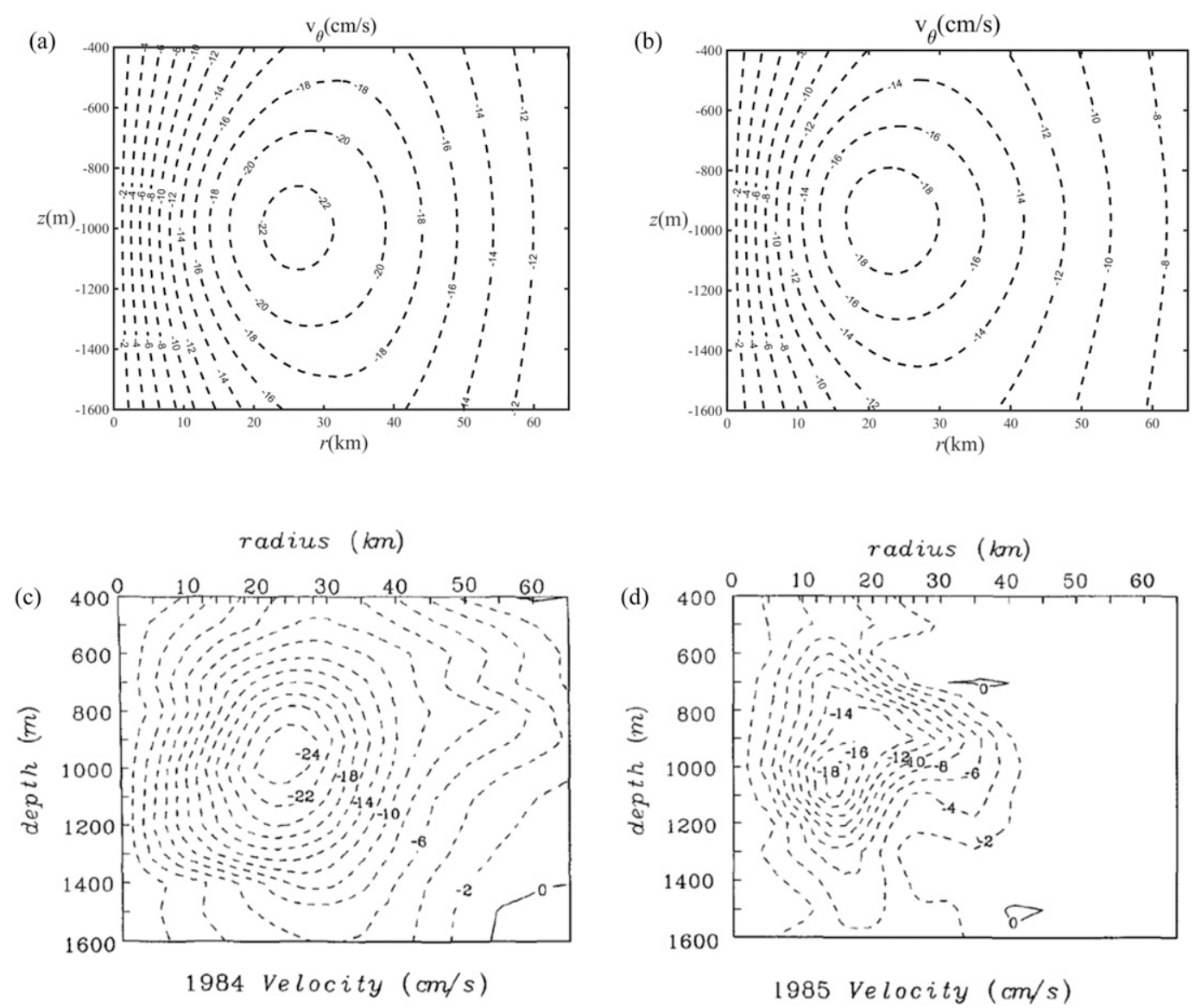

FIG. 9. (a),(b) The $(r, z)$ patterns of the azimuthal velocity $v_{\theta}$ for the PV-Gaussian models in (16) and (17). The observationally inferred sections (from Schultz Tokos and Rossby 1991) across Meddy Sharon taken in (c) 1984 and (d) 1985 are presented.

$$
\mathrm{PV}=\mathrm{PV}_{0}\left\{1-0.7 \exp \left[-\frac{r^{2}}{r_{0}^{2}}-\frac{\left(z-z_{0}\right)^{2}}{h^{2}}\right]\right\}
$$

where, as previously (section 3 ), $r_{0}=30 \mathrm{~km}, z_{0}=$ $-1000 \mathrm{~m}$, and $h=40 \mathrm{~m}$. The azimuthal velocity $v_{\theta}$ and density $\rho$ patterns were iteratively reconstructed using the procedure described in Sutyrin and Radko (2016). Yet another initialization (EXP10) assumed the Gaussian $\mathrm{PV}$ pattern in the isopycnal $(r, \rho)$ system:

$$
\mathrm{PV}=\mathrm{PV}_{0}\left\{1-0.7 \exp \left[-\frac{r^{2}}{r_{0}^{2}}-\frac{\left(\rho-\rho_{0}\right)^{2}}{(\Delta \rho)^{2}}\right]\right\},
$$

where $\Delta \rho=0.076 \mathrm{~kg} \mathrm{~m}^{-3}$. Both models (16) and (17) were characterized by the monotonic increase of PV with the distance from the meddy axis, which held promise of their dynamic stability. To demonstrate that these models are qualitatively consistent with observations, in Fig. 9 we present the $(r, z)$ patterns of azimuthal velocities used in EXP9 (Fig. 9a) and EXP10 (Fig. 9b) along with the sections across Meddy Sharon from the field programs conducted in 1984 (Fig. 9c) and in 1985

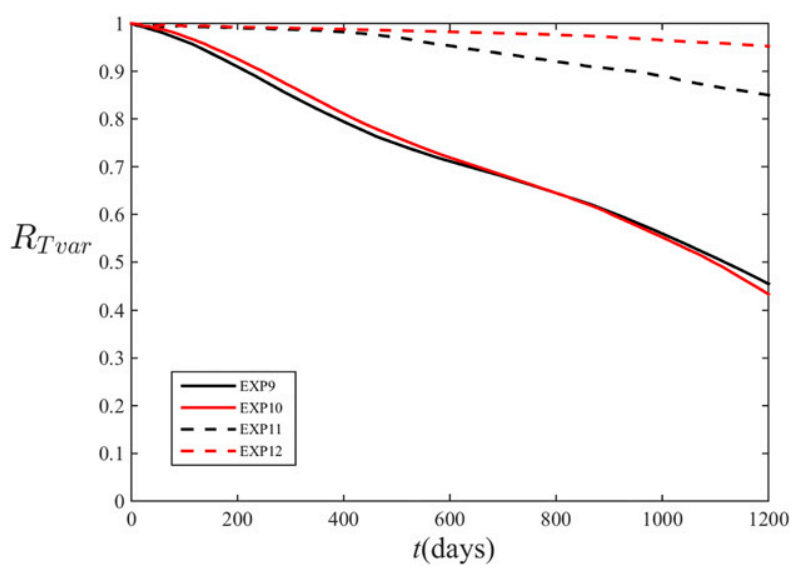

FIG. 10. As in Fig, 6, but for EXP9-EXP12. 
(a)

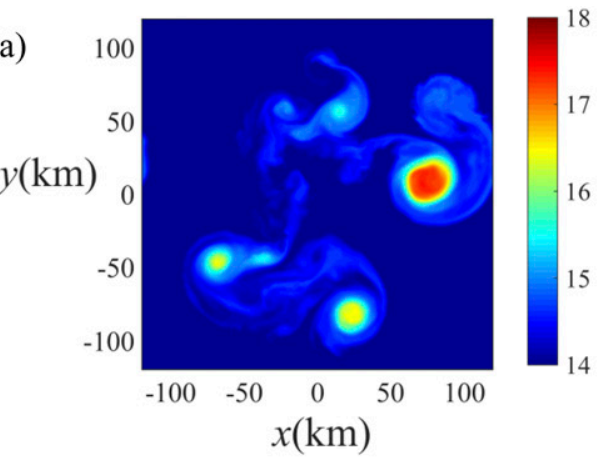

(b)

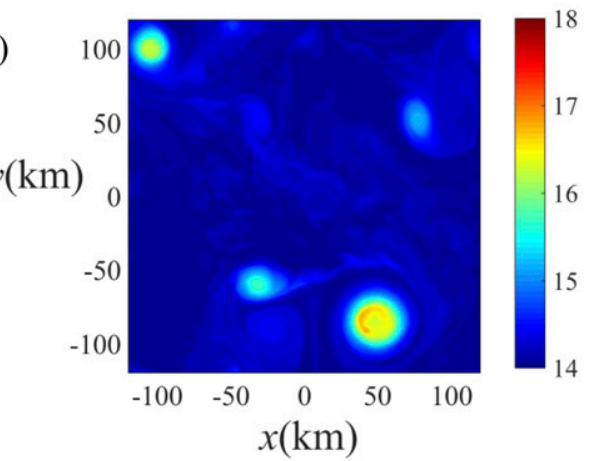

(c)

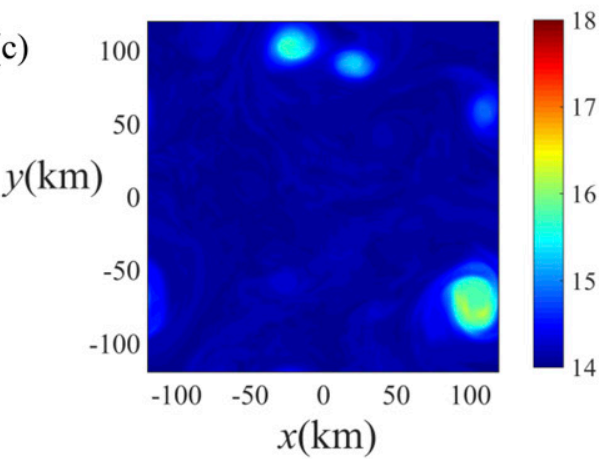

(d)

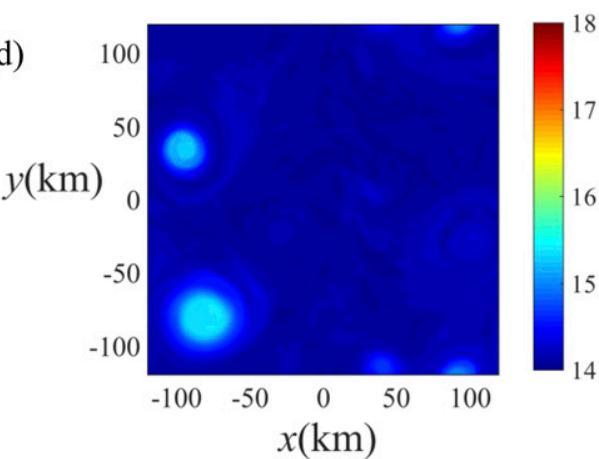

(e)

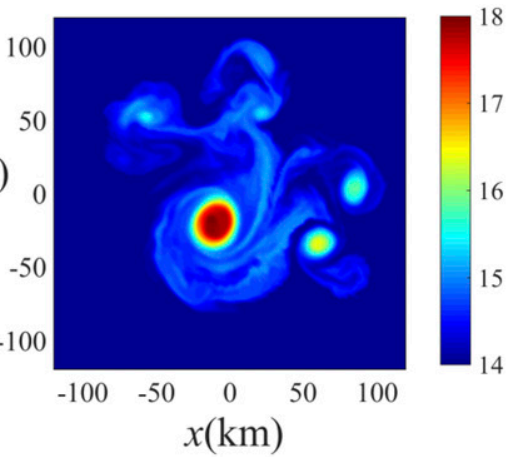

(f)

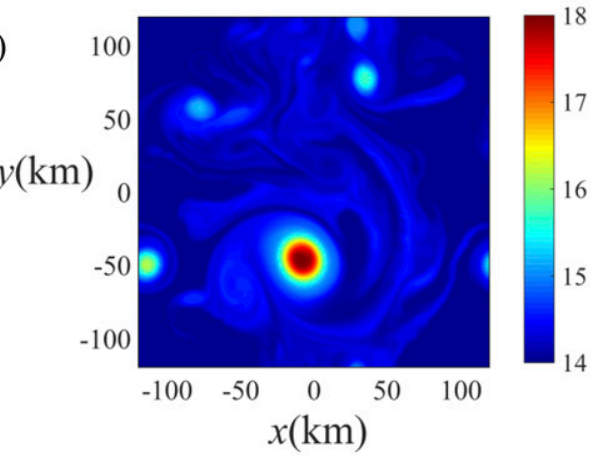

(g)

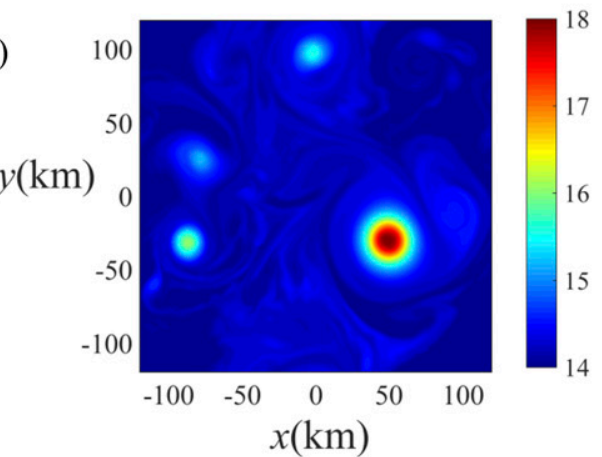

(h)

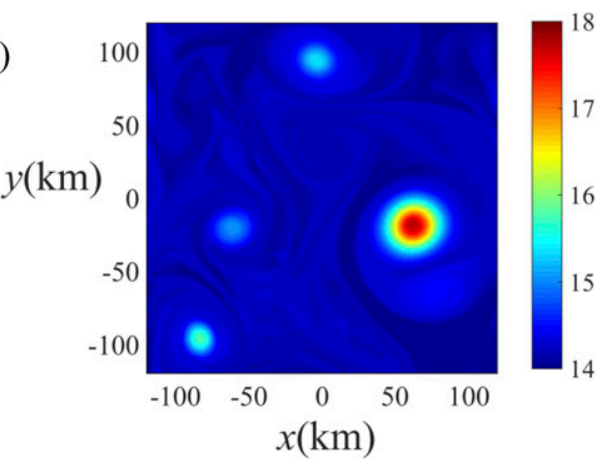

FIG. 11. The evolution of the unstable Gaussian meddy. The horizontal temperature sections $T(x, y)$ at $z=-1000 \mathrm{~m}$ in the double-diffusive simulation for $t=$ (a) 250, (b) 500, (c) 750, and (d) 1000 days. (e)-(h) The corresponding sections in the non-double-diffusive run. 


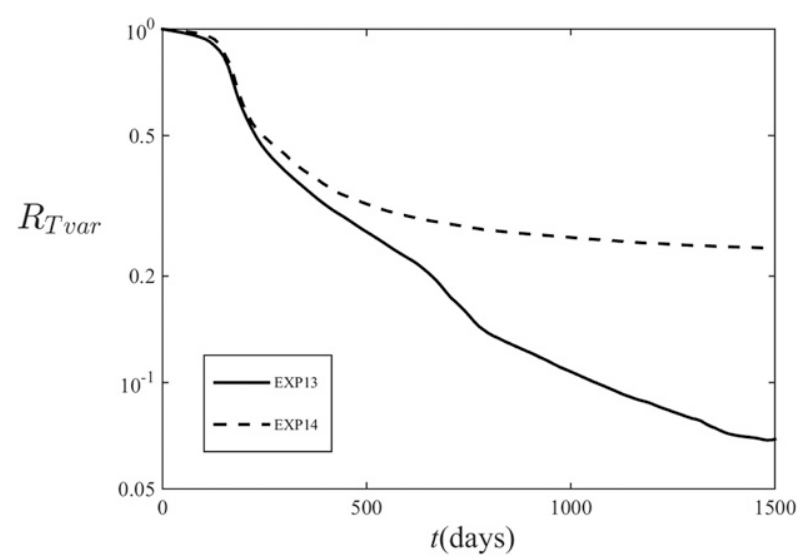

FIG. 12. The diagnostic variable $R_{\text {Tvar }}$ on the logarithmic scale as a function of time for EXP13 and EXP14.

(Fig. 9d). Overall, the structures in Fig. 9 are mutually consistent in terms of the location of maximal velocity $r_{\mathrm{v} \max } \approx(15-24) \mathrm{km}$ and its value $\left|v_{\max }\right|=(0.18-0.24)$ $\mathrm{cm} \mathrm{s}^{-1}$. Theoretical solutions, however, are characterized by a more gradual decay of the velocity field in the meddy periphery $(r>30 \mathrm{~km})$.

To evaluate the role of thermohaline interleaving in meddy dispersion, in Fig. 10 we plot our key diagnostic variable $R_{\text {Tvar }}$ as a function of time for simulations EXP9 and EXP10 along with their non-double-diffusive counterparts (EXP11 and EXP12). The results in Fig. 10 are consistent with our earlier findings (section 3 ). With double diffusion, the meddy decay occurs on the time scale of approximately 3 years, which is broadly consistent with observational estimates (e.g., Ruddick et al. 2010). Without double diffusion (EXP11 and EXP12), the meddy dispersion is substantially slower. It is interesting that EXP11 exhibits some signs of dynamic instability, despite the monotonicity of its PV distribution (16). This instability is not entirely unexpected since theoretical stability proofs for monotonic PV patterns have only been developed for effectively unbounded (horizontally and vertically) domains and for quasigeostrophic models (Dritschel 1988). This leaves open the possibility that our numerical setup may not exactly satisfy the stability conditions. Fortunately, the instability of the meddy in EXP11 is rather benign. It does not lead to vortex fragmentation and, in the course of experiment ( $t=1200$ days), reduces the perturbation temperature variance by a mere $15 \%$ (Fig. 10).

Our final examples (EXP13 and EXP14) illustrate the evolution of a strongly unstable vortex with and without double diffusion. The initial condition for the following simulations is based on the Gaussian vortex [(1)], which was previously used in the design of a stable meddy (section 3). While these experiments may not be directly relevant to meddies, which are known for their ability to remain coherent for years, they contribute to our general understanding of the interaction between mesoscale turbulence and interleaving. The first stage of both experiments is characterized by the fragmentation of the initial Gaussian vortex into smaller vortices (Fig. 11). This process is completed within the first year and is accompanied by the transfer of the meddy available potential energy into kinetic energy [see the discussion in Sutyrin and Radko (2016)]. As a result, the isopycnal surfaces flatten and the perturbation temperature variance reduces by a factor of 3 . During the fragmentation period $(t<500$ days), simulations EXP13 and EXP14 exhibit qualitatively similar patterns (Figs. 11a,e), and the corresponding time records of the diagnostic variable $R_{\text {Tvar }}$ (Fig. 12) barely deviate from each other. However, major differences become apparent in the late evolutionary stage $(t>500$ days). The non-doublediffusive experiment (EXP14) evolves to a statistically steady regime, characterized by nearly constant $R_{\mathrm{Tvar}}$. In contrast, $R_{\text {Tvar }}$ in EXP13 continues to decrease, and, by the end of the experiment ( $t=1500$ days), it is less than in its non-double-diffusive counterpart (EXP14) by a factor of 3.5 (Fig. 12). The range of temperature values at the meddy center plane $(z=-1000 \mathrm{~m})$ is also strongly affected by interleaving (Fig. 11). In EXP14, the temperature range remains fairly uniform throughout the entire simulation period (Figs. 11e-h), whereas in the double-diffusive simulation (EXP13) it reduces from $4^{\circ} \mathrm{C}$ initially to $1.46^{\circ} \mathrm{C}$ at $t=1500$ days (Figs. $11 \mathrm{a}-\mathrm{d}$ ). All this evidence consistently points to a potentially critical role of thermohaline interleaving in the ultimate dispersion of mesoscale turbulence.

\section{Discussion}

This study presents the first series of intrusionresolving simulations of intrathermocline vortices exemplified by meddies, anticyclonic lenses of Mediterranean origin, commonly found in the lower North Atlantic thermocline. These simulations are used to validate and quantify the earlier, observationally inspired theories of meddy dispersion, which invoked thermohaline interleaving as the dominant mechanism for the erosion of their thermohaline signatures. The diagnostics of our numerical experiments yielded the following conclusions:

- Simulations confirm that double-diffusive interleaving is indeed an essential dissipative process, controlling the longevity and evolutionary patterns of the observed intrathermocline eddies. In the model runs without intrusions, life-spans of meddies greatly 
exceed the observationally inferred time scales. In contrast, incorporating double diffusion into the model formulation results in realistic erosion rates that are consistent with oceanographic measurements.

- The effective lateral diffusivity of temperature diagnosed from simulations $\left(K_{H}=1.6 \mathrm{~m}^{2} \mathrm{~s}^{-1}\right)$ conforms to-but is biased toward the lower end of - the observationally inferred range of $K_{H}=1-5 \mathrm{~m}^{2} \mathrm{~s}^{-1}$ (e.g., Ruddick et al. 2010). The overall agreement implies that that the current computational capabilities, as well as our ability to represent small-scale mixing processes, have advanced sufficiently to start producing consistent intrusion-resolving models of meso- and basin-scale phenomena.

- The perceived bias toward low lateral diffusivities in simulations could be attributed either to observational uncertainties or to the overly aggressive representation of diapycnal shear-induced mixing by the assumed KPP scheme. The latter possibility motivates further refinement and validation of mixing models (e.g., Smyth et al. 2002) specifically tailored for the intrusion dynamics.

- The background turbulence has a profoundly adverse effect on the meddy dispersion. Under normal circumstances, mechanically generated turbulence acts to intensify the diapycnal dispersion of thermal anomalies. However, this direct contribution to meddy erosion is greatly outweighed by the tendency of turbulence to suppress interleaving and thus to indirectly limit the lateral dispersion and extend the meddy life-span.

- Major differences were observed in the dynamics and erosion rates of the rapidly rotating meddies, which more accurately represent observed structures, and their density-compensated quiescent counterparts. The longevity of baroclinic meddies is attributed to their higher susceptibility to Kelvin-Helmholtz instabilities, which tend to suppress interleaving and the associated lateral erosion. In this regard, it should be mentioned that the majority of theoretical and laboratory intrusion studies focused on densitycompensated background states (e.g., Ruddick and Turner 1979; Ruddick et al. 1999; Krishnamurti 2006; Simeonov and Stern 2007, 2008; Radko 2011). Undoubtedly, these investigations have brought much insight into the dynamics of interleaving. However, our findings suggest that density-compensated models should be applied to oceanic phenomena with caution since baroclinic shears, which are ubiquitous in nature, could dramatically affect the intensity and mixing characteristics of intrusions.
It should be emphasized that the present study is not meant to offer an exhaustive analysis of the dispersion mechanisms of intrathermocline vortices. Several potentially important effects have been intentionally excluded in order to isolate the dynamics of interaction between meddies and intrusions. For instance, it would be interesting to analyze the meddy dispersion in the presence of beta effect and basin-scale shears. The decision to prioritize the investigation of interleaving at this stage is motivated by one of the most fundamental and long-standing challenges in geophysical fluid dynamics - the explanation of the direct cascade of energy and thermal variance. The mechanical and thermodynamic forcing at the sea surface occurs on the scales of ocean basins, whereas the energy and thermal variance are ultimately dissipated by molecular processes acting on the microscale (Wunsch and Ferrari 2004; Merryfield 2005). Interleaving explicitly connects microstructure with much larger scales of motion, and therefore investigations of its properties may lead to critical insights into the cascade problem. Our results show that interleaving can be highly effective in dissipating mesoscale variability. This finding supports the proposition (Garrett 1982; Ruddick and Kerr 2003; Song et al. 2011) that the link between mesoscale stirring and microscale mixingthe least understood connection in the cascade theorymay indeed involve thermohaline interleaving.

Acknowledgments. The authors thank the editor Karen Heywood and the anonymous reviewers for helpful comments. Support of the National Science Foundation (Grant OCE-1334914) is gratefully acknowledged.

\section{REFERENCES}

Ambar, I., N. Serra, M. Brogueira, G. Cabecadas, F. Abrantes, P. Freitas, C. Goncalves, and N. Gonzalez, 2002: Physical, chemical and sedimentological aspects of the Mediterranean outflow off Iberia. Deep-Sea Res. II, 49, 4163-4177, https:// doi.org/10.1016/S0967-0645(02)00148-0.

Armi, L., D. Hebert, N. Oakey, J. Price, P. Richardson, T. Rossby, and B. Ruddick, 1988: The movements and decay of a Mediterranean salt lens. Nature, 333, 649-651, https://doi.org/ 10.1038/333649a0.

$-,-\longrightarrow,-,-,-$, and,- 1989 : Two years in the life of a Mediterranean salt lens. J. Phys. Oceanogr., 19, 354-370, https://doi.org/10.1175/1520-0485(1989)019<0354: TYITLO $>2.0 . C O ; 2$.

Beckers, M., R. Verzicco, H. J. H. Clercx, and G. J. F. van Heijst, 2001: Dynamics of pancake-like vortices in a stratified fluid: Experiments, model and numerical simulations. J. Fluid Mech., 433, 1-27, https://doi.org/10.1017/ S0022112001003482.

Benilov, E. S., 2005: On the stability of ocean vortices: A solution to the problem? Dyn. Atmos. Oceans, 40, 133-149, https:// doi.org/10.1016/j.dynatmoce.2004.10.017. 
— and J. D. Flanagan, 2008: The effect of ageostrophy on the stability of vortices in a two-layer ocean. Ocean Modell., 23, 49-58, https://doi.org/10.1016/j.ocemod.2008.03.004.

Carton, X. J., 2001: Hydrodynamical modeling of oceanic vortices. Surv. Geophys., 22, 179-263, https://doi.org/10.1023/A: 1013779219578.

D'Asaro, E. A., 1988: Observations of small eddies in the Beaufort Sea. J. Geophys. Res., 93, 6669-6684, https://doi.org/10.1029/ JC093iC06p06669.

Dewar, W. K., and P. D. Killworth, 1995: On the stability of oceanic rings. J. Phys. Oceanogr., 25, 1467-1487, https://doi.org/ 10.1175/1520-0485(1995)025<1467:OTSOOR >2.0.CO;2.

- J. R. Blundell, and P. D. Killworth, 1999: Primitive equation instability of wide oceanic rings. Part II: Numerical studies of ring stability. J. Phys. Oceanogr., 29, 1744-1785, https://doi.org/ 10.1175/1520-0485(1999)029<1744:PEIOWO > 2.0.CO;2.

Dritschel, D. G., 1988: Nonlinear stability bounds for inviscid, twodimensional, parallel or circular flows with monotonic vorticity, and the analogous three-dimensional quasi-geostrophic flows. J. Fluid Mech., 191, 575-581, https://doi.org/10.1017/ S0022112088001715.

Elliott, B. A., and T. B. Sanford, 1986: The subthermocline lens D1. Part II: Kinematics and dynamics. J. Phys. Oceanogr., 16, 549-561, https://doi.org/10.1175/1520-0485(1986)016<0549: TSLDPI $>2.0 . \mathrm{CO} ; 2$

Garrett, C., 1982: On the parameterization of diapycnal fluxes due to double-diffusive intrusions. J. Phys. Oceanogr., 12, 952-959, https://doi.org/10.1175/1520-0485(1982)012<0952: OTPODF $>2.0 . \mathrm{CO} ; 2$.

Hebert, D., N. Oakey, and B. R. Ruddick, 1990: Evolution of a Mediterranean salt lens: Scalar properties. J. Phys. Oceanogr., 20, 1468-1483, https://doi.org/10.1175/1520-0485(1990)020<1468: EOAMSL $>2.0 . \mathrm{CO} ; 2$.

Howard, L. N., 1961: Note on a paper of John W. Miles. J. Fluid Mech., 10, 509-512, https://doi.org/10.1017/ S0022112061000317.

Joyce, T. M., 1977: A note on the lateral mixing of water masses. J. Phys. Oceanogr., 7, 626-629, https://doi.org/10.1175/ 1520-0485(1977)007<0626:ANOTLM>2.0.CO;2.

- W. Zenk, and J. M. Toole, 1978: The anatomy of the Antarctic Polar Front in the Drake Passage. J. Geophys. Res., 83, 6093-6113, https://doi.org/10.1029/JC083iC12p06093.

Krishnamurti, R., 2006: Double-diffusive interleaving on horizontal gradients. J. Fluid Mech., 558, 113-131, https://doi.org/ 10.1017/S002211200600992X.

Kuzmina, N., and V. Zhurbas, 2000: Effects of double diffusion and turbulence on interleaving at baroclinic oceanic fronts. J. Phys. Oceanogr., 30, 3025-3038, https://doi.org/10.1175/ 1520-0485(2000)030<3025:EODDAT > 2.0.CO;2.

Large, W. G., J. C. McWilliams, and S. C. Doney, 1994: Oceanic vertical mixing: A review and a model with a nonlocal boundary layer parameterization. Rev. Geophys., 32, 363-403, https://doi.org/10.1029/94RG01872.

Marshall, J., A. Adcroft, C. Hill, L. Perelman, and C. Heisey, 1997a: A finite-volume, incompressible Navier Stokes model for studies of the ocean on parallel computers. J. Geophys. Res., 102, 5753-5766, https://doi.org/10.1029/96JC02775.

C. Hill, L. Perelman, and A. Adcroft, 1997b: Hydrostatic, quasi-hydrostatic, and nonhydrostatic ocean modeling. J. Geophys. Res., 102, 5733-5752, https://doi.org/10.1029/ 96JC02776.

May, B. D., and D. E. Kelley, 1997: Effect of baroclinicity on double-diffusive interleaving. J. Phys. Oceanogr., 27,
1997-2008, https://doi.org/10.1175/1520-0485(1997)027<1997: EOBODD $>2.0 . \mathrm{CO} ; 2$.

McDowell, S. E., and H. T. Rossby, 1978: Mediterranean water: An intense mesoscale eddy off the Bahamas. Science, 202, 1085-1087, https://doi.org/10.1126/science.202.4372.1085.

Merryfield, W. J., 2005: Ocean mixing in 10 steps. Science, 308, 641-642, https://doi.org/10.1126/science.1111417.

Miles, J. W., 1961: On the stability of heterogeneous shear flows. J. Fluid Mech., 10, 496-508, https://doi.org/10.1017/ S0022112061000305.

Mueller, R. D., W. D. Smyth, and B. Ruddick, 2007: Shear and convective turbulence in a model of thermohaline intrusions. J. Phys. Oceanogr., 37, 2534-2549, https://doi.org/10.1175/JPO3137.1.

Nguyen, H. Y., B. L. Hua, R. Schopp, and X. Carton, 2012: Slow quasigeostrophic unstable modes of a lens vortex in a continuously stratified flow. Geophys. Astrophys. Fluid Dyn., 106, 305-319, https://doi.org/10.1080/03091929.2011.620568.

Osborn, T. R., and C. S. Cox, 1972: Oceanic fine structure. Geophys. Fluid Dyn., 3, 321-345, https://doi.org/10.1080/ 03091927208236085.

Paillet, J., B. Le Cann, X. Carton, Y. Morel, and A. Serpette, 2002: Dynamics and evolution of a northern meddy. J. Phys. Oceanogr., 32, 55-79, https://doi.org/10.1175/1520-0485(2002)032<0055: DAEOAN $>2.0 . \mathrm{CO} ; 2$.

Pingree, R. D., and B. Le Cann, 1993: Structure of a meddy (Bobby 92) southeast of the Azores. Deep-Sea Res. I, 40, 2077-2103, https://doi.org/10.1016/0967-0637(93)90046-6.

Prater, M. D., and T. B. Sanford, 1994: A meddy off Cape St. Vincent. Part I: Description. J. Phys. Oceanogr., 24, 1572-1586, https:// doi.org/10.1175/1520-0485(1994)024<1572:AMOCSV>2.0.CO;2.

Radko, T., 2003: A mechanism for layer formation in a doublediffusive fluid. J. Fluid Mech., 497, 365-380, https://doi.org/ 10.1017/S0022112003006785.

_ 2011: Mechanics of thermohaline interleaving: Beyond the empirical flux laws. J. Fluid Mech., 675, 117-140, https:// doi.org/10.1017/S0022112011000061.

—, 2013: Double-Diffusive Convection. Cambridge University Press, 342 pp.

— diffusive convection. J. Fluid Mech., 692, 5-27, https://doi.org/ 10.1017/jfm.2011.343.

Rayleigh, L., 1879: On the stability, or instability, of certain fluid motions. Proc. London Math. Soc., 11, 57-70, https://doi.org/ 10.1112/plms/s1-11.1.57.

Richardson, L. F., 1920: The supply of energy from and to atmospheric eddies. Proc. Roy. Soc. London, A97, 354-373, https:// doi.org/10.1098/rspa.1920.0039.

Ruddick, B., 1992: Intrusive mixing in a Mediterranean salt lens-Intrusion slopes and dynamical mechanisms. $J$. Phys. Oceanogr., 22, 1274-1285, https://doi.org/10.1175/ 1520-0485(1992)022<1274:IMIAMS > 2.0.CO;2.

— diffusive intrusions. Deep-Sea Res., 26A, 903-913, https:// doi.org/10.1016/0198-0149(79)90104-3.

, and D. Hebert, 1988: The mixing of Meddy 'Sharon.' Small-Scale Turbulence and Mixing in the Ocean, J. Nihoul and B. Jamart, Eds., Elsevier Oceanography Series, Vol. 46, Elsevier, 249-262. , and O. Kerr, 2003: Oceanic thermohaline intrusions: Theory. Prog. Oceanogr., 56, 483-497, https://doi.org/10.1016/ S0079-6611(03)00029-6.

— Observations. Prog. Oceanogr., 56, 499-527, https://doi.org/ 10.1016/S0079-6611(03)00028-4. 
—, O. M. Phillips, and J. S. Turner, 1999: A laboratory and quantitative model of finite-amplitude thermohaline intrusions. Dyn. Atmos. Oceans, 30, 71-99, https://doi.org/ 10.1016/S0377-0265(99)00021-4.

_ N. N. Oakey, and D. Hebert, 2010: Measuring lateral heat flux across a thermohaline front: A model and observational test. J. Mar. Res., 68, 523-539, https://doi.org/10.1357/ 002224010794657146

Schultz Tokos, K., and T. Rossby, 1991: Kinematics and dynamics of a Mediterranean salt lens. J. Phys. Oceanogr., 21, 879-892, https://doi.org/10.1175/1520-0485(1991)021<0879: $\mathrm{KADOAM}>2.0 . \mathrm{CO} ; 2$.

Simeonov, J., and M. E. Stern, 2007: Equilibration of two-dimensional double-diffusive intrusions. J. Phys. Oceanogr., 37, 625-643, https://doi.org/10.1175/JPO3000.1.

—, and —, 2008: Double-diffusive intrusions in a stable salinity gradient "heated from below." J. Phys. Oceanogr., 38, 2271-2282, https://doi.org/10.1175/2008JPO3913.1.

Smyth, W. D., 2008: Instabilities of a baroclinic, double-diffusive frontal zone. J. Phys. Oceanogr., 38, 840-861, https://doi.org/ 10.1175/2007JPO3770.1.

- , and B. Ruddick, 2010: Effects of ambient turbulence on interleaving at a baroclinic front. J. Phys. Oceanogr., 40, 685-712, https://doi.org/10.1175/2009JPO4297.1.

—, E. Skyllingstad, G. Crawford, and H. Wijesekera, 2002: Nonlocal fluxes and Stokes drift effects in the K-profile parameterization. Ocean Dyn., 52, 104-115, https://doi.org/ 10.1007/s10236-002-0012-9.

Sokolovskiy, M. A., and J. Verron, 2014: Dynamics of Vortex Structures in a Stratified Rotating Fluid. Atmospheric and Oceanographic Sciences Library Series, Vol. 47, Springer, 382 pp.

Song, H., L. M. Pinheiro, B. Ruddick, and F. C. Teixeira, 2011: Meddy, spiral arms, and mixing mechanisms viewed by seismic imaging in the Tagus Abyssal Plain (SW Iberia). J. Mar. Res., 69, 827-842, https://doi.org/10.1357/002224011799849309.

Stellmach, S., A. Traxler, P. Garaud, N. Brummell, and T. Radko, 2011: Dynamics of fingering convection. Part 2: The formation of thermohaline staircases. J. Fluid Mech., 677, 554-571, https:// doi.org/10.1017/jfm.2011.99.

Stern, M. E., 1967: Lateral mixing of water masses. Deep-Sea Res. Oceanogr. Abstr., 14, 747-753, https://doi.org/10.1016/ S0011-7471(67)80011-1.

Sutyrin, G., and T. Radko, 2016: Stabilization of isolated vortices in a rotating stratified fluid. Fluids, 1, 26, https://doi.org/ 10.3390/fluids1030026.

Turner, J. S., 1978: Double-diffusive intrusions into a density gradient. J. Geophys. Res., 83, 2887-2901, https://doi.org/10.1029/ JC083iC06p02887.

Tychensky, A., and X. Carton, 1998: Hydrological and dynamical characterization of meddies in the Azores region: A paradigm for baroclinic vortex dynamics. J. Geophys. Res., 103, 25 061-25 079, https://doi.org/10.1029/97JC03418.

Washburn, L., and R. H. Käse, 1987: Double diffusion and the distribution of the density ratio in the Mediterranean waterfront southeast of the Azores. J. Phys. Oceanogr., 17, 12-25, https:// doi.org/10.1175/1520-0485(1987)017<0012:DDATDO > 2.0.CO;2.

Wunsch, C., and R. Ferrari, 2004: Vertical mixing, energy, and the general circulation of the oceans. Annu. Rev. Fluid Mech., 36, 281-314, https://doi.org/10.1146/annurev.fluid.36.050802.122121.

Yim, E., P. Billant, and C. Menesguen, 2016: Stability of an isolated pancake vortex in continuously stratified-rotating fluids. J. Fluid Mech., 801, 508-553, https://doi.org/10.1017/jfm.2016.402.

Zhurbas, V., and I. S. Oh, 2001: Can turbulence suppress doublediffusively driven interleaving completely? J. Phys. Oceanogr., 31, 2251-2254, https://doi.org/10.1175/1520-0485(2001)031<2251: CTSDDD $>2.0 . \mathrm{CO} ; 2$ 